TRANSACTIONS OF THE

AMERICAN MATHEMATICAL SOCIETY

Volume 364, Number 1, January 2012, Pages 1-29

S 0002-9947(2011)05216-5

Article electronically published on August 11, 2011

\title{
THE SPECTRUM OF SCHRÖDINGER OPERATORS AND HODGE LAPLACIANS ON CONFORMALLY CUSP MANIFOLDS
}

\author{
SYLVAIN GOLÉNIA AND SERGIU MOROIANU
}

\begin{abstract}
We describe the spectrum of the $k$-form Laplacian on conformally cusp Riemannian manifolds. The essential spectrum is shown to vanish precisely when the $k$ and $k-1$ de Rham cohomology groups of the boundary vanish. We give Weyl-type asymptotics for the eigenvalue-counting function in the purely discrete case. In the other case we analyze the essential spectrum via positive commutator methods and establish a limiting absorption principle. This implies the absence of the singular spectrum for a wide class of metrics. We also exhibit a class of potentials $V$ such that the Schrödinger operator has compact resolvent, although in most directions the potential $V$ tends to $-\infty$. We correct a statement from the literature regarding the essential spectrum of the Laplacian on forms on hyperbolic manifolds of finite volume, and we propose a conjecture about the existence of such manifolds in dimension 4 whose cusps are rational homology spheres.
\end{abstract}

\section{INTRODUCTION}

There exist complete, non-compact manifolds on which the scalar Laplacian has purely discrete spectrum; see e.g. 9. The goal of this paper is to understand such phenomena for the Laplacian on differential forms in a more geometric setting. We aim to provide eigenvalue asymptotics whenever the spectrum is purely discrete and to clarify the nature of the essential spectrum when it arises.

We study $n$-dimensional non-compact Riemannian manifolds $\left(X, g_{p}\right)$ which outside a compact set are diffeomorphic to a cylinder $(0, \infty) \times M$, where $M$ is a closed, possibly disconnected Riemannian manifold. The metric on $X$ near infinity is assumed to be a perturbation of the model metric

$$
g_{p}^{\text {model }}=y^{-2 p}\left(d y^{2}+h\right), \quad y \gg 0
$$

where $h$ is a metric on $M$ independent of $y$, and $p>0$. For $p=1, g_{p}^{\text {model includes }}$ the case of finite-volume complete hyperbolic manifolds, which we discuss in some

Received by the editors December 8, 2008 and, in revised form, September 29, 2009.

2000 Mathematics Subject Classification. Primary 58J40, $58 \mathrm{Z} 05$.

Key words and phrases. Finite volume hyperbolic manifolds, Hodge theory, Laplacian on forms, cusp pseudodifferential operators, purely discrete spectrum, absolutely continuous spectrum, Mourre estimate.

The authors were partially supported by the contract MERG 006375, funded through the European Commission.

The second author was partially supported from the contracts 2-CEx06-11-18/2006 and CNCSIS-GR202/19.09.2006. 
detail in the last section of the paper. For $p>1$, the metric (1.1) is of metrichorn type as in [24]. The general form of the Riemannian metric $g_{p}$ is given in (2.1). It is in particular quasi-isometric to the model metric (1.1), in the sense that there exist positive constants $A, B$ such that for every tangent vector $v$, we have $A\|v\|_{g_{p}} \leq\|v\|_{g_{p}^{\text {model }}} \leq B\|v\|_{g_{p}}$. Moreover $g_{p}$ must admit a full asymptotic expansion as $x \rightarrow 0$. The metric $g_{p}$ is incomplete if and only if $p>1$, and $\operatorname{vol}\left(X, g_{p}\right)<\infty$ if and only if $p>1 / n$.

We denote by $\Delta=d^{*} d+\delta^{*} \delta$ the Hodge Laplacian defined on smooth forms with compact support in $X$. It is a symmetric non-negative operator in $L^{2}\left(X, \Lambda^{*} X, g_{p}\right)$, and we also denote by $\Delta$ its self-adjoint Friedrichs extension. If $p \leq 1$, i.e. if $\left(X, g_{p}\right)$ is complete, then $\Delta$ is essentially self-adjoint; see [11. Since $\Delta$ preserves the space of $k$-forms, we can define $\Delta_{k}$ as its restriction to $\Lambda^{k} X$, which is also self-adjoint.

Let us advertise one application of our results. The essential spectrum of the Laplacian acting on forms on non-compact manifolds has been extensively studied, as it provides information on the Hodge decomposition of the space of $L^{2}$-forms. Without attempting to give an exhaustive bibliography, we mention here the papers [6, 8, 17, 26, 27, 28. In Section 7, we show:

Proposition 1.1. Let $(X, g)$ be a complete non-compact hyperbolic manifold of finite volume. Let $M$ be the boundary at infinity. Let $\tilde{g}$ be the metric $(1+\rho) g$, with $\rho \in \mathcal{C}^{\infty}(X, \mathbb{R}), \inf (\rho)>-1$ such that

$$
\rho(x) \rightarrow 0 \text {, as } x \rightarrow M \text { and }\|d \rho\|_{\infty}<\infty .
$$

If $n=\operatorname{dim}(X)$ is odd, also suppose that the Betti number $b_{\frac{n-1}{2}}(M)$ vanishes. Then $\operatorname{Im}(d)$ and $\operatorname{Im}\left(\delta_{\tilde{g}}\right)$ are closed, and

$$
L^{2}\left(X, \Lambda^{*} X, \tilde{g}\right)=\operatorname{ker}\left(\Delta_{\tilde{g}}\right) \oplus \operatorname{Im}(d) \oplus \operatorname{Im}\left(\delta_{\tilde{g}}\right) .
$$

This proposition follows from the fact that the essential spectrum $\sigma_{\text {ess }}(\Delta)$ of $\Delta$ does not contain 0 . For hyperbolic manifolds of finite volume, $\sigma_{\text {ess }}(\Delta)$ was computed by Mazzeo and Phillips [28. Theorem 1.11], but their statement contains a gap; see Section 7 for geometric counterexamples. In [3], Antoci also computes $\sigma_{\text {ess }}\left(\Delta_{k}\right)$ but for technical reasons she is unable to decide whether 0 is isolated in the essential spectrum of $\Delta_{k}$ except in the case where $M=S^{n-1}$ with the standard metric. For the metric (1.1) and for a general $M$, we compute $\sigma_{\text {ess }}\left(\Delta_{k}\right)$ in Proposition 5.2 and we deduce that 0 is never isolated in it for any $k$. In the Appendix, we also give a self-contained proof of the stability of the essential spectrum of the Laplacian acting on forms for a large class of perturbations of the metric.

In this paper we first investigate the absence of the essential spectrum for $\Delta_{k}$ and improve the results of [3] along the way. We replace the condition $M=S^{n-1}$ with a weaker topological condition on the boundary at infinity $M$. Related results were obtained in [5, 16, 32. The following theorem holds for the conformally cusp metric (2.1), a generalization of (1.1). To our knowledge, the result on eigenvalue asymptotics in this context is entirely new.

Theorem 1.2. Let $X$ be an n-dimensional conformally cusp manifold (Definition 2.1) for some $p>0$. Fix an integer $k$ between 0 and $n$. If the Betti numbers $b_{k}(M)$ and $b_{k-1}(M)$ of the boundary at infinity $M$ both vanish, then:

(1) the Laplacian $\Delta_{k}$ acting on $k$-forms on $X$ is essentially self-adjoint in $L^{2}$ for the metric $g_{p}$;

(2) the spectrum of $\Delta_{k}$ is purely discrete; 
(3) the asymptotic of its eigenvalues, in the limit $\lambda \rightarrow \infty$, is given by

$$
N_{p}(\lambda) \approx \begin{cases}C_{1} \lambda^{n / 2} & \text { for } 1 / n<p, \\ C_{2} \lambda^{n / 2} \log \lambda & \text { for } p=1 / n \\ C_{3} \lambda^{1 / 2 p} & \text { for } 0<p<1 / n\end{cases}
$$

Note that the hypothesis $b_{k}(M)=b_{k-1}(M)=0$ does not hold for $k=0,1$; it also does not hold for $k=n, n-1$ if $M$ has at least one orientable connected component. In particular, Theorem 1.2 does not apply to the Laplacian acting on functions. We refer to Section 7 for a discussion of this hypothesis, implications about hyperbolic manifolds and open problems in dimension 4 and higher.

The constants $C_{1}, C_{2}$ are given by (3.4), (3.5). Up to a universal constant, which only depends on $\operatorname{dim}(X)$, they are just the volume of $\left(X, g_{p}\right)$ in the finite volume case $p>1 / n$ (here we get the precise form of the Weyl law for closed manifolds), respectively the volume of the boundary at infinity $M$ with respect to a naturally induced metric in the case $p=1 / n$. When the metric $g_{0}$ is exact (see Definition 2.2. this includes the model metric (1.1) ), $C_{3}$ is given by (3.7). While $C_{2}$ may be viewed as the diverging term in a regularizing process for the volume of $\left(X, g_{p}\right), C_{3}$ is essentially a spectral zeta function on $M$ evaluated at the point $1 / p-1$, which belongs to the domain of convergence since $1 / p-1>\operatorname{dim}(M)=n-1$. It is unclear whether this constant also represents some renormalized volume of $g_{p}$.

We stress that $\Delta_{k}$ is essentially self-adjoint and has purely discrete spectrum based solely on the hypothesis $b_{k}(M)=b_{k-1}(M)=0$, without any such condition as completeness of the metric or finiteness of the volume; see Corollary 3.2 . Intuitively, the continuous spectrum of $\Delta_{k}$ is governed by zero-modes of the form Laplacian on $M$ in dimensions $k$ and $k-1$ (both dimensions are involved because of algebraic relations in the exterior algebra). By Hodge theory, the kernel of the $k$-form Laplacian on the compact manifold $M$ is isomorphic to $H^{k}(M)$; hence these zero-modes (harmonic forms) exist precisely when the Betti numbers do not vanish. We remark that, in the study of the scalar magnetic Laplacian [16] and of the Dirac operator [5], the rôle of the Betti numbers was played by an integrality condition on the magnetic field, respectively by a topological condition on the spin structure on the cusps.

We are now interested in some refined (and less studied) properties of the essential spectrum, namely the absence of a singularly continuous spectrum and weighted estimates of the resolvent, which give non-trivial dynamical properties on the group $e^{i t \Delta_{k}}$. For the model metric (1.1), in the complete case, a refined analysis was started in 2. We determine the nature of the essential spectrum by positive commutator techniques. The case of the Laplacian on functions has been treated originally by this method in [10, and by many other methods in the literature; see for instance [19, 22] for different approaches. In [16, we introduced a conjugate operator which was "local in energy", in order to deal with a larger class of perturbations of the model metric. We use the same idea here; however, the analysis of the Laplacian on $k$-forms turns out to be more involved than that of the scalar magnetic Laplacian. Indeed, one could have two thresholds and the positivity is harder to extract between them. The difficulty arises from the compact part of the manifold, since we can diagonalize the operator only on the cusp ends. The resolvent of the operator does not stabilize this decomposition. To deal with 
this, we introduce a perturbation of the Laplacian which uncouples the compact part from the cusps in a gentle way.

Let $L$ be the operator on $\mathcal{C}_{c}^{\infty}\left(X, \Lambda^{*} X\right)$ of multiplication by a fixed smooth function $L: X \rightarrow[1, \infty)$ defined by:

$$
L(y):=\left\{\begin{array}{ll}
\ln (y) & \text { for } p=1, \\
\frac{y^{1-p}}{1-p} & \text { for } p<1,
\end{array} \quad \text { for } y \geq y_{0} \text { and for some } y_{0} \geq 1 .\right.
$$

Given $s \geq 0$, let $\mathscr{L}_{s}$ be the domain of $L^{s}$ equipped with the graph norm. We set $\mathscr{L}_{-s}:=\mathscr{L}_{s}^{*}$, where the adjoint space is defined so that $\mathscr{L}_{s} \subset L^{2}\left(X, \Lambda^{*} X, g_{p}\right) \subset \mathscr{L}_{s}^{*}$, using the Riesz lemma. Given $I \subset \mathbb{R}$, let $I_{ \pm}$be the set of complex numbers $a \pm i b$, where $a \in I$ and $b>0$.

Perturbations of short-range (resp. long-range) type are denoted with the subscript sr (resp. lr); they are supported in $(2, \infty) \times M$. We ask long-range perturbations to be radial. In other words, a perturbation $W_{\text {lr }}$ satisfies $W_{\operatorname{lr}}(y, m)=$ $W_{\text {lr }}\left(y, m^{\prime}\right)$ for all $m, m^{\prime} \in M$.

Theorem 1.3. Let $\varepsilon>0$. We consider the metric $\tilde{g}=\left(1+\rho_{\mathrm{sr}}+\rho_{\mathrm{lr}}\right) g_{p}^{\text {model }}$, with $0<p \leq 1$ and where the short-range and long-range components satisfy

$$
\begin{gathered}
L^{1+\varepsilon} \rho_{\mathrm{sr}}, d \rho_{\mathrm{sr}} \text { and } \Delta_{g} \rho_{\mathrm{sr}} \in L^{\infty}(X), \\
L^{\varepsilon} \rho_{\mathrm{lr}}, L^{1+\varepsilon} d \rho_{\mathrm{lr}} \text { and } \Delta_{g} \rho_{\mathrm{lr}} \in L^{\infty}(X) .
\end{gathered}
$$

Suppose that at least one of the two Betti numbers $b_{k}(M)$ and $b_{k-1}(M)$ is non-zero. Let $V=V_{\mathrm{loc}}+V_{\mathrm{sr}}$ and $V_{\mathrm{lr}}$ be some potentials, where $V_{\mathrm{loc}}$ is measurable with compact support and $\Delta_{k}$-compact, and $V_{\mathrm{sr}}$ and $V_{\mathrm{lr}}$ are in $L^{\infty}(X)$ such that:

$$
\left\|L^{1+\varepsilon} V_{\mathrm{sr}}\right\|_{\infty}<\infty, V_{\mathrm{lr}} \rightarrow 0 \text {, as } y \rightarrow+\infty \text { and }\left\|L^{1+\varepsilon} d V_{\mathrm{lr}}\right\|_{\infty}<\infty \text {. }
$$

Consider the Schrödinger operators $H_{0}=\Delta_{k, p}+V_{\mathrm{lr}}$ and $H=H_{0}+V$. Then

(1) The essential spectrum of $H$ is $[\inf \{\kappa(p)\}, \infty)$, where the set of thresholds $\kappa(p) \subset \mathbb{R}$ is defined in (5.2), and is not empty.

(2) $H$ has no singular continuous spectrum.

(3) The eigenvalues of $H$ have finite multiplicity and no accumulation points outside $\kappa(p)$.

(4) Let $\mathcal{J}$ be a compact interval such that $\mathcal{J} \cap\left(\kappa(p) \cup \sigma_{\mathrm{pp}}(H)\right)=\emptyset$. Then, for all $s$ in $\left(\frac{1}{2}, \frac{3}{2}\right)$, there exists $c$ such that

$$
\left\|\left(H-z_{1}\right)^{-1}-\left(H-z_{2}\right)^{-1}\right\|_{\mathcal{B}\left(\mathscr{L}_{s}, \mathscr{L}_{-s}\right)} \leq c\left|z_{1}-z_{2}\right|^{s-1 / 2},
$$

for all $z_{1}, z_{2} \in \mathcal{J}_{ \pm}$.

(5) Let $\mathcal{J}=\mathbb{R} \backslash \kappa(p)$ and let $E_{0}$ and $E$ be the continuous spectral components of $H_{0}$ and $H$, respectively. Then, the wave operators defined as the strong limit

$$
\Omega_{ \pm}=\operatorname{s-lim}_{t \rightarrow \pm \infty} e^{i t H} e^{-i t H_{0}} E_{0}(\mathcal{J})
$$

exist and are complete; i.e. $\Omega_{ \pm} \mathscr{H}=E(\mathcal{J}) \mathscr{H}$, where $\mathscr{H}=L^{2}\left(X, \Lambda^{k} X, \tilde{g}\right)$.

Although Theorem 1.3 is stated only for conformal perturbations of the model metric, the statement (11) remains true for a wide family of metrics asymptotic to (1.1); see Proposition 5.2. The fact that every eigenspace is finite-dimensional (in particular, for the possible eigenvalues contained in the set of thresholds $\kappa(p)$ ) is due to the general result [16, Lemma B.1] and holds for an arbitrary conformally cusp metric (2.1). The proof of the rest, (2)-(5) where in (3) we consider eigenvalues with 
energy different from $\kappa(p)$, relies on the Mourre theory [1, 33] with an improvement for the regularity of the boundary value of the resolvent; see [13] and the references therein. These wide classes of perturbations of the metric have been introduced in [16]; however, here the treatment of long-range perturbations is different from the one in [16. We prove these facts in Section 6.4. Note that this general class of metrics seems to be difficult to analyze by standard scattering techniques.

We now turn to the question of the perturbation of the Laplacian $\Delta_{k}$ by some non-relatively compact potential. In the Euclidean $\mathbb{R}^{n}$, using Persson's formula, one sees that $\sigma_{\text {ess }}(H)$ is empty for $H=\Delta+V$ if $V \in L_{\text {loc }}^{\infty}$ tends to $\infty$ at infinity. However, the converse is wrong as noted in [39] by taking $V\left(x_{1}, x_{2}\right)=x_{1}^{2} x_{2}^{2}$, which gives rise to a compact resolvent. Morally speaking, a particle cannot escape in the direction of finite energy at infinity which is too narrow compared to the very attracting part of $V$ which tends to infinity. In our setting, the space being smaller at infinity, it is easier to create this type of situation even if most of the potential tends to $-\infty$. To our knowledge, the phenomenon is new. The general statement appears in Proposition 4.1 .

Proposition 1.4. Let $p>0$ and $\left(X, g_{p}\right)$ be a conformally cusp manifold. Let $V$ in $y^{2 p} \mathcal{C}^{\infty}(\bar{X})$ be a smooth potential with Taylor expansion $y^{-2 p} V=V_{0}+y^{-1} V_{1}+$ $O\left(y^{-2}\right)$ at infinity. Assume that $V_{0}$ is non-negative and not identically zero on any connected component of $M$. Then the Schrödinger operator $\Delta_{k}+V$ is essentially self-adjoint and has purely discrete spectrum. The eigenvalues obey the generalized Weyl law (1.3) and the constants $C_{1}, C_{2}$ do not depend on $V$.

As in Theorem 1.2. the completeness of the manifold is not required to obtain the essential self-adjointness of the operator. Note that by assuming $p>1 / 2$ (in particular, on finite-volume hyperbolic manifolds, for which $p=1$ ) and $V_{1}<0$, we get $V \sim y^{2 p-1} V_{1}$ tends to $-\infty$ as we approach $M \backslash \operatorname{supp}\left(V_{0}\right)$. The support of the non-negative leading term $V_{0}$ must be non-empty, but otherwise it can be chosen arbitrarily small.

Some of the results about the essential spectrum appeared in the unpublished paper [15].

Notation. Given two Hilbert spaces $\mathscr{H}$ and $\mathscr{K}$, we denote by $\mathcal{B}(\mathscr{H}, \mathscr{K})$ the space of bounded operators from $\mathscr{H}$ to $\mathscr{K}$, and by $\mathcal{K}(\mathscr{H}, \mathscr{K})$ the subspace of compact operators. When $\mathscr{K}=\mathscr{H}$, we simply write $\mathcal{B}(\mathscr{H})$ and $\mathcal{K}(\mathscr{H})$. Given a closed operator $H$ acting in $\mathscr{H}$, we denote by $\mathcal{D}(H)$ its domain and we endow it with the graph norm $\|\cdot\|+\|H \cdot\|$. We denote by $\sigma(H)$ its spectrum and by $\rho(H)$ its resolvent set. Given a vector bundle $E$ over a smooth manifold $X$, we denote by $\mathcal{C}_{c}^{\infty}(X, E)$ the space of smooth sections in $E$ with compact support.

\section{Geometric Definitions}

Let $\bar{X}$ be a smooth $n$-dimensional compact manifold with closed boundary $M$, and $x: \bar{X} \rightarrow[0, \infty)$ a boundary-defining function. Let $\mathcal{I} \subset \mathcal{C}^{\infty}(\bar{X})$ be the principal ideal generated by the function $x$. A cusp vector field is a smooth vector field $V$ on $\bar{X}$ such that $d x(V) \in \mathcal{I}^{2}$. The space ${ }^{c} \mathcal{V}$ of cusp vector fields forms a Lie subalgebra ${ }^{c} \mathcal{V}$ of the Lie algebra of smooth vector fields on $\bar{X}$. Moreover, there exists a smooth vector bundle ${ }^{c} T \bar{X} \rightarrow \bar{X}$ whose space of sections identifies naturally with ${ }^{c} \mathcal{V}$.

A cusp metric on $\bar{X}$ is a (smooth) Euclidean metric $g_{0}$ on the bundle ${ }^{c} T \bar{X}$ over $\bar{X}$. Since ${ }^{c} T \bar{X}$ and $T X$ are canonically identified over the interior $X:=\bar{X} \backslash M, g_{0}$ 
induces a complete Riemannian metric on $X$. We want to study the Laplacian on $k$-forms associated to the metric $g_{p}:=x^{2 p} g_{0}$ for fixed $p>0$.

Fix a product decomposition of $\bar{X}$ near $M$, i.e., an embedding $[0, \varepsilon) \times M \hookrightarrow \bar{X}$ compatible with the function $x$. Then near the boundary, $g_{p}$ and the cusp metric $g_{0}$ take the form

$$
g_{0}=a\left(\frac{d x}{x^{2}}+\alpha(x)\right)^{2}+h(x), \quad g_{p}:=x^{2 p} g_{0}, \quad p>0,
$$

where $a \in \mathcal{C}^{\infty}(\bar{X})$ such that $a_{\mid M}>0, h$ is a smooth family of symmetric 2-tensors on $M$ and $\alpha$ is a smooth family of 1 -forms in $\mathcal{C}^{\infty}\left([0, \varepsilon) \times M, \Lambda^{1}(M)\right)$. Note that the model metric (1.1) is a particular case of the metric $g_{p}$ from (2.1) with $\alpha \equiv 0, a \equiv 1$ and $h(x)$ constant in $x$ (to see this, set $x:=1 / y$ in (1.1)).

Definition 2.1. A Riemannian manifold $\left(X, g_{p}\right)$ which is the interior of a compact manifold with boundary together with a Riemannian metric $g_{p}$ of the form (2.1) near the boundary for some $p>0$ is called a conformally cusp manifold. The boundary $M=\partial X$ may be disconnected.

Note that $g_{p}$ is quasi-isometric to the model metric $g_{p}^{\text {model }}$ from (1.1), but not every metric quasi-isometric to $g_{p}^{\text {model }}$ is conformally cusp in the sense of the above definition.

By [32, Lemma 6], the function $a_{0}:=a(0)$, the metric $h_{0}:=h(0)$ and the class modulo exact forms of the 1 -form $\alpha_{0}:=\alpha(0)$, defined on $M$, are independent of the chosen product decomposition and of the boundary-defining function $x$ inside the fixed cusp structure. We also recall the following definition.

Definition 2.2. The metric $g_{0}$ is called exact if $a_{0}=1$ and $\alpha_{0}$ is an exact 1-form.

Let $E, F \rightarrow \bar{X}$ be smooth vector bundles. The space of cusp differential operators $\operatorname{Diff}_{c}(\bar{X}, E, F)$ is the space of those differential operators which in local trivializations can be written as the composition of cusp vector fields and smooth bundle morphisms. The normal operator of $P \in \operatorname{Diff}_{c}(\bar{X}, E, F)$ is defined by

$$
\mathbb{R} \ni \xi \mapsto \mathcal{N}(P)(\xi):=\left(e^{i \xi / x} P e^{-i \xi / x}\right)_{\mid x=0} \in \operatorname{Diff}\left(M, E_{\mid M}, F_{\mid M}\right) .
$$

From the definition, $\operatorname{ker} \mathcal{N}=\mathcal{I}$. Diff ${ }_{c}$, which we denote again by $\mathcal{I}$.

Example 2.3. Given a family $\left(P_{x}\right)_{x \in[0, \epsilon)}$ of operators on $M$ depending smoothly on $x$, one has $\mathcal{N}\left(P_{x}\right)(\xi)=P_{0}$. Also, $\mathcal{N}\left(x^{2} \partial_{x}\right)(\xi)=i \xi$.

From the definition, the normal operator map is linear and multiplicative. Let $P \in \operatorname{Diff}_{c}(\bar{X}, E, F)$ be a cusp operator and $P^{*}$ its adjoint with respect to $g_{0}$. Then $\mathcal{N}\left(P^{*}\right)(\xi)$ is the adjoint of $\mathcal{N}(P)(\xi)$ for the volume form $a_{0}{ }^{1 / 2} d h_{0}$ and with respect to the metric on $E_{\mid M}, F_{\mid M}$ induced by restriction. Indeed, since $\mathcal{N}$ commutes with products and sums, it is enough to check the claim for the set of local generators of Diff ${ }_{c}$ from Example 2.3, which is a straightforward computation.

\section{Proof of Theorem 1.2}

We follow the ideas of 32 and [16. We will first show that $\Delta_{k}$ is $x^{-2 p}$ times an elliptic cusp differential operator. Since we work on bundles, we first trivialize the bundles of forms in the $x$-direction. Near $M$, set

$$
V_{0}:=x^{2} \partial_{x} \in{ }^{c} \mathcal{V}, \quad V^{0}:=x^{-2} d x+\alpha(x) .
$$


We get an orthogonal decomposition of smooth vector bundles

$$
\Lambda^{k}\left({ }^{c} T \bar{X}\right) \simeq \Lambda^{k}(T M) \oplus V^{0} \wedge \Lambda^{k-1}(T M),
$$

where $\Lambda^{*}(T M)$ is identified with the kernel of the contraction by $V_{0}$.

The de Rham differential $d: \mathcal{C}^{\infty}\left(X, \Lambda^{k} X\right) \rightarrow \mathcal{C}^{\infty}\left(X, \Lambda^{k+1} X\right)$ restricts to a cusp differential operator $d: \mathcal{C}^{\infty}\left(\bar{X}, \Lambda^{k}\left({ }^{c} T \bar{X}\right)\right) \rightarrow \mathcal{C}^{\infty}\left(\bar{X}, \Lambda^{k+1}\left({ }^{c} T \bar{X}\right)\right)$. Its normal operator in the decomposition (3.2) is

$$
\mathcal{N}(d)(\xi)=\left[\begin{array}{cc}
d^{M}-i \xi \alpha_{0} \wedge & d^{M} \alpha_{0} \wedge \\
i \xi & -\left(d^{M}-i \xi \alpha_{0} \wedge\right)
\end{array}\right] .
$$

The principal symbol of a cusp operator in $\operatorname{Diff}_{c}^{*}(\bar{X}, E, F)$ extends as a map on the cusp cotangent bundle. An operator $P \in x^{-2 p} \operatorname{Diff}_{c}^{*}(\bar{X}, E, F)$ is called cuspelliptic in the sense of Melrose if the principal symbol of $x^{2 p} P$ is invertible on ${ }^{c} T^{*} \bar{X} \backslash\{0\}$ down to $x=0$; it is called fully elliptic if it is cusp-elliptic and if the differential operator $\mathcal{N}\left(x^{2 p} P\right)(\xi)$ on $M$ is invertible as an unbounded operator in $L^{2}\left(M, E_{\mid M}, F_{\mid M}\right)$ for all $\xi \in \mathbb{R}$; see [29].

Proposition 3.1. The Laplacian $\Delta_{k}$ of the metric $g_{p}$ belongs to

$$
x^{-2 p} \operatorname{Diff}_{c}^{2}\left(\bar{X}, \Lambda^{k}\left({ }^{c} T \bar{X}\right)\right)
$$

and is cusp-elliptic. Moreover, $x^{2 p} \Delta_{k}$ is fully elliptic if and only if the de Rham cohomology groups $H_{\mathrm{dR}}^{k}(M)$ and $H_{\mathrm{dR}}^{k-1}(M)$ both vanish.

Proof. The principal symbol of the Laplacian of $g_{p}$ on $\Lambda^{k} X$ is $g_{p}$ times the identity. Since $x^{-2 p} g_{p}=g_{0}$ on the cotangent bundle, and since $g_{0}$ extends by definition to a positive-definite bilinear form on ${ }^{c} T^{*} \bar{X}$, it follows that $x^{2 p} \Delta_{k}$ is cusp-elliptic. Let $\delta_{0}^{k}, \delta_{p}^{k}$ be the formal adjoint of $d: \Lambda^{k} X \rightarrow \Lambda^{k+1}(X)$ with respect to $g_{0}$, resp. $g_{p}$. Then $\delta_{p}^{k}=x^{(2 k-n) p+2} \delta_{0} x^{(n-2(k+1)) p-2}$. By conjugation invariance of the normal operator, we obtain $\mathcal{N}\left(x^{2 p}\left(d \delta_{p}+\delta_{p} d\right)\right)=\mathcal{N}\left(d \delta_{0}+\delta_{0} d\right)$. By Hodge theory, the kernel of $\mathcal{N}\left(d \delta_{0}+\delta_{0} d\right)(\xi)$ is isomorphic to the cohomology of the complex $\left(\Lambda^{*}\left({ }^{c} T \bar{X}\right)_{\mid M}, \mathcal{N}(d)(\xi)\right)$. We write

$$
\mathcal{N}(d)(\xi)=A(\xi)+B(\xi),
$$

where

$$
A(\xi)=\left[\begin{array}{cc}
0 & 0 \\
i \xi & 0
\end{array}\right], B(\xi)=\left[\begin{array}{cc}
d^{M}-i \xi \alpha_{0} \wedge & d^{M} \alpha_{0} \wedge \\
0 & -\left(d^{M}-i \xi \alpha_{0} \wedge\right)
\end{array}\right] .
$$

We claim that for $\xi \neq 0$ the cohomology of $\mathcal{N}(d)(\xi)$ vanishes. The idea is to again use Hodge theory but with respect to the volume form $d h_{0}$ on $M$. Then $B(\xi)^{*}$ anti-commutes with $A(\xi)$ and similarly $A(\xi)^{*} B(\xi)+B(\xi) A(\xi)^{*}=0$. Therefore

$$
\mathcal{N}(d)(\xi) \mathcal{N}(d)(\xi)^{*}+\mathcal{N}(d)(\xi)^{*} \mathcal{N}(d)(\xi)=\xi^{2} I+B(\xi)^{*} B(\xi)+B(\xi) B(\xi)^{*},
$$

where $I$ is the $2 \times 2$ identity matrix. So for $\xi \neq 0$ the Laplacian of $\mathcal{N}(d)(\xi)$ is a strictly positive elliptic operator; hence it is invertible.

Let us turn to the case $\xi=0$. We claim that the cohomology of $\left(\Lambda^{*} M \oplus\right.$ $\left.\Lambda^{*-1} M, \mathcal{N}(d)(0)\right)$ is isomorphic to $H_{\mathrm{dR}}^{*}(M) \oplus H_{\mathrm{dR}}^{*-1}(M)$. Indeed, notice that

$$
\mathcal{N}(d)(0)=\left[\begin{array}{cc}
1 & -\alpha_{0} \wedge \\
0 & 1
\end{array}\right]\left[\begin{array}{cc}
d^{M} & 0 \\
0 & -d^{M}
\end{array}\right]\left[\begin{array}{cc}
1 & \alpha_{0} \wedge \\
0 & 1
\end{array}\right] .
$$

In other words, the differential $\mathcal{N}(d)(0)$ is conjugated to the diagonal de Rham differential, so they have isomorphic cohomology. 
Corollary 3.2. If $b_{k}(M)=b_{k-1}(M)=0$, then for every $p>0$, the Laplacian $\Delta_{k}$ of the metric $g_{p}$ is essentially self-adjoint on $\mathcal{C}_{c}^{\infty}\left(X, \Lambda^{k} X\right)$ and has purely discrete spectrum. The domain of its self-adjoint extension is the weighted cusp Sobolev space $x^{2 p} H_{c}^{2}\left(X, \Lambda^{k} X\right)$.

Note that for $p \leq 1$ the metric $g_{p}$ is complete, in which case $\Delta_{k}$ is essentially selfadjoint without any extra hypothesis; see [11. However, even in the complete case, we do not know how to describe the domain of the unique self-adjoint extension if the Betti numbers do not vanish. The definition of the cusp Sobolev spaces is recalled in the proof below.

Proof. By the de Rham theorem, the vanishing of the Betti numbers is equivalent to the vanishing of the de Rham cohomology groups $H_{\mathrm{dR}}^{k}(M)$ and $H_{\mathrm{dR}}^{k-1}(M)$. Hence from Proposition 3.1 it follows that $x^{2 p} \Delta_{k}$ is fully elliptic. From the general properties of the cusp calculus [30, there exists a Green operator $G \in x^{2 p} \Psi_{c}^{-2}\left(X, \Lambda^{k} X\right)$ which inverts $\Delta_{k}$ up to remainders in the ideal $x^{\infty} \Psi_{c}^{-\infty}\left(X, \Lambda^{k} X\right)$. Although we do not use it here, recall that $\Psi_{c}^{k}(X, E, F)$ is defined as a space of distributional kernels on $X \times X$ (with coefficients in the bundle $E \otimes F^{*}$ ) which are classically conormal to the diagonal and with prescribed asymptotics near the boundary. More precisely, they must lift to be extendible across the front face of a double blow-up resolution of the corner $M \times M$ in $\bar{X} \times \bar{X}$, and to vanish in Taylor series at the other boundary hyperfaces. We refer e.g. to 32 for the precise definition. Once the properties of the calculus have been established, the existence of the Green operator $G$ is a standard application of elliptic theory.

The Sobolev space $H_{c}^{q}\left(X, \Lambda^{k} X\right)$ is by definition the intersection of the domains of the maximal extensions of all elliptic cusp operators inside $\Psi_{c}^{q}\left(X, \Lambda^{k} X\right)$. Cusp operators of order $r \in \mathbb{C}$ map $H_{c}^{q}(X)$ to $H_{c}^{q-\Re(r)}(X)$; see [32].

Now look at $\Delta_{k}$ restricted to $\mathcal{C}_{c}^{\infty}\left(X, \Lambda^{k} X\right)$. It is easy to see that $x^{2 p} H_{c}^{2}(X)$ is contained in the domain of the minimal extension of $\Delta_{k}$. Conversely, using the Green operator $G$ and the mapping properties of cusp operators stated above, we see that every vector in the domain of the maximal extension of $\Delta_{k}$ belongs to $x^{2 p} H_{c}^{2}(X)$. In conclusion, the minimal and the maximal domain are the same and equal to $x^{2 p} H_{c}^{2}(X)$.

Now recall from 32 that for $p, q>0$, operators in $x^{p} \Psi_{c}^{-q}(X)$ are compact. Since the self-adjoint operator $\Delta_{k}$ has a compact inverse modulo compact operators, it follows that it has a purely discrete spectrum.

Notice that we have more generally proved that a symmetric fully elliptic cusp operator of order $(-p,-r)$ with $p, r>0$ on a conformally cusp manifold is essentially self-adjoint and has a purely discrete spectrum.

We can now conclude the proof of Theorem 1.2. By Proposition 3.1, if we assume that $b_{k}(M)=b_{k-1}(M)=0$ it follows that $\Delta_{k}$ is fully elliptic. From Corollary 3.2 . this implies (by a form of elliptic regularity) that $\Delta_{k}$ is essentially self-adjoint and the domain of the extension is the weighted Sobolev space $x^{2 p} H^{2}(X)$. By [32, Theorem 17], the spectrum of $\Delta_{k}$ is purely discrete and accumulates towards infinity according to (1.3), modulo identification of the correct coefficients. This is proved in two steps as in 32]: first, the complex powers of a self-adjoint fully elliptic cusp operator belong again to the cusp calculus and form an analytic family; secondly, the trace of an analytic family in the complex variable $z$ of cusp operators of order $(-z,-p z)$ is well-defined for $z<-n, p z<-1$, and extends to a meromorphic 
function on $\mathbb{C}$ with at most double poles at certain reals. By [32, Proposition 14] and Delange's theorem ([32, Lemma 16]), the coefficients $C_{1}, C_{2}, C_{3}$ are determined by the order of the first occurring pole of the zeta function (i.e., the smallest $z \in \mathbb{R}$ which is a pole for $\zeta(z):=\operatorname{Tr}\left(\Delta_{k}^{-z / 2}\right)$ and by its leading coefficient in the Laurent expansion. The principal symbol $\sigma_{1}\left(\Delta_{k}^{1 / 2}\right)$ is identically 1 on the cosphere bundle. The dimension of the form bundle equals the binomial coefficient $\left(\begin{array}{l}n \\ k\end{array}\right)$.

3.1. The case $p>1 / n$. From [32, Proposition 14], the first pole of $\zeta$ is simple, located at $z=n$ with residue

$$
R_{1}=(2 \pi)^{-n}\left(\begin{array}{l}
n \\
k
\end{array}\right) \operatorname{vol}(X) \operatorname{vol}\left(S^{n-1}\right) .
$$

From [32, Lemma 16], we get the asymptotic equivalence for the eigenvalues of $\Delta_{k}^{1 / 2}$ :

$$
N\left(\Delta_{k}^{1 / 2}, \lambda\right) \approx \frac{R_{1}}{n} \lambda^{n} .
$$

Taking into account $N\left(\Delta_{k}^{1 / 2}, \lambda^{1 / 2}\right)=N\left(\Delta_{k}, \lambda\right)$, we get

$$
C_{1}=\left(\begin{array}{l}
n \\
k
\end{array}\right) \frac{\operatorname{Vol}\left(X, g_{p}\right) \operatorname{Vol}\left(S^{n-1}\right)}{n(2 \pi)^{n}} .
$$

3.2. The case $p=1 / n$. From [32, Proposition 14], the first pole of $\zeta$ is double, located at $z=n$ with leading coefficient

$$
R_{2}=n(2 \pi)^{-n}\left(\begin{array}{l}
n \\
k
\end{array}\right) \operatorname{vol}(M) \operatorname{vol}\left(S^{n-1}\right) .
$$

From [32, Lemma 16], the eigenvalues of $\Delta_{k}^{1 / 2}$ obey

$$
N\left(\Delta_{k}^{1 / 2}, \lambda\right) \approx \frac{R_{2}}{n} \lambda^{n} \log \lambda
$$

Again translating from the counting function of $\Delta_{k}^{1 / 2}$ to that of $\Delta_{k}$, we get

$$
C_{2}=\left(\begin{array}{l}
n \\
k
\end{array}\right) \frac{\operatorname{Vol}\left(M, h_{0}\right) \operatorname{Vol}\left(S^{n-1}\right)}{2(2 \pi)^{n}} .
$$

3.3. The case $p<1 / n$. In this situation the first pole of $\zeta$ is simple, located at $z=1 / p$ with residue

$$
-\frac{n}{2 \pi} \int_{\mathbb{R}} \operatorname{Tr} \mathcal{N}\left(x^{-1} \Delta_{k}^{-\frac{1}{2 p}}\right)(\xi) d \xi
$$

To compute $C_{3}$, we also suppose that the metric $g_{0}$ is exact. With this assumption, by replacing $x$ with another boundary-defining function inside the same cusp structure, we can assume that $\alpha_{0}=0$ (see [32]). In this case, (3.3) gives

$$
\mathcal{N}\left(x^{2 p} \Delta_{k}\right)(\xi)=\left[\begin{array}{cc}
\xi^{2}+\Delta_{k}^{M} & 0 \\
0 & \xi^{2}+\Delta_{k-1}^{M}
\end{array}\right] .
$$

This allows us to compute the integral from [32, Proposition 14] in terms of the zeta functions of the Laplacians on forms on $M$ with respect to $h_{0}$. Straightforwardly, 
one gets

$$
C_{3}=\frac{\Gamma\left(\frac{1-p}{2 p}\right)\left(\zeta\left(\Delta_{k}^{M}, \frac{1}{p}-1\right)+\zeta\left(\Delta_{k-1}^{M}, \frac{1}{p}-1\right)\right)}{2 \sqrt{\pi} \Gamma\left(\frac{1}{2 p}\right)} .
$$

This ends the proof of Theorem 1.2

\section{SCHRÖDINGER OPERATORS AND DISCRETE SPECTRUM}

In this section, we prove the compactness of the resolvent of the Schrödinger operator for a class of potentials that tend to $+\infty$ only towards a very small part of the infinity. Proposition 1.4 for the metric (2.1) is a particular case of this analysis.

Let $H_{0}$ be a cusp pseudodifferential operator on $X$. We say that $H_{0}$ has the unique continuation property at infinity if for all $\xi \in \mathbb{R}$, the normal operator $\mathcal{N}\left(H_{0}\right)(\xi)$ has the (weak) unique continuation property as an operator on each connected component of $M$; i.e., the non-zero solutions $\phi$ to the pseudodifferential equation $\mathcal{N}\left(H_{0}\right)(\xi) \phi=0$ do not vanish on any open set.

Proposition 4.1. Let $g_{p}$ be the metric on $X$ given by (2.1) near $\partial X$. Let $H_{0}$ be a non-negative cusp-elliptic operator, $H_{0} \in x^{-q p} \Psi_{c}^{q}(X, E)$ for some $q>0$. Assume that $x^{q p} H_{0}$ has the unique continuation property at infinity. Let $V$ be a self-adjoint potential in $x^{-q p} \mathcal{C}^{\infty}(\bar{X}, E)$. Assume $V_{0}:=\left(x^{q p} V\right)_{\mid M} \in \mathcal{C}^{\infty}\left(M, E_{\mid M}\right)$ is semipositive definite and in each connected component of $M$ there is $z$ with $V_{0}(z)>0$. Then $H:=H_{0}+V$ is essentially self-adjoint in $L^{2}(X, E)$ and $\sigma_{\mathrm{ess}}(H)=\emptyset$. Its eigenvalue-counting function, as $\lambda$ goes to infinity, satisfies

$$
N_{H}(\lambda) \approx \begin{cases}C_{1}^{\prime} \lambda^{n / q} & \text { for } 1 / n<p<\infty, \\ C_{2}^{\prime} \lambda^{n / q} \log \lambda & \text { for } p=1 / n, \\ C_{3}^{\prime} \lambda^{\frac{1}{q p}} & \text { for } p<1 / n .\end{cases}
$$

Proof. We start by proving that the operator $H$ is fully elliptic. Let $\xi \in \mathbb{R}$. A nonzero solution $\phi$ of $\mathcal{N}\left(x^{q p} H\right)(\xi) \phi=0$ satisfies $\left\langle\mathcal{N}\left(x^{q p} H_{0}\right)(\xi) \phi, \phi\right\rangle+\left\langle V_{0} \phi, \phi\right\rangle=0$. The operators $\mathcal{N}\left(x^{q p} H_{0}\right)(\xi)$ and $V_{0}$ being non-negative, we get $\mathcal{N}\left(x^{q p} H_{0}\right)(\xi) \phi=0$ and $V_{0} \phi=0$. By unique continuation, solutions of the elliptic operator $\mathcal{N}\left(x^{q p} H_{0}\right)(\xi)$ which are not identically zero on a given connected component of $M$ do not vanish on any open subset of that component. However since $V_{0} \phi=0$ and $V_{0}(z)>0, \phi$ must vanish in the neighborhood of $z$ where $V_{0}$ is invertible, a contradiction. Thus $H$ is fully elliptic.

By [32, Lemma 10 and Corollary 13], it follows that $H$ is essentially self-adjoint with domain $x^{q p} H_{c}^{q}(M)$, and has purely discrete spectrum. By [32, Proposition 14], the constants $C_{1}^{\prime}$ and $C_{2}^{\prime}$ can be computed as in Section 2 . They depend only on the principal symbol of $H$ and so they are independent of $V$. The coefficient $C_{3}^{\prime}$ depends only on $\mathcal{N}(H)$.

The unique continuation property holds for instance when $\mathcal{N}\left(H_{0}\right)(\xi)$ is an elliptic second-order differential operator for all $\xi$, in particular for the Laplacians $\Delta_{k}$ on differential forms or the (scalar) magnetic Laplacian, as in [16]. Thus Proposition 4.1 applies to $\Delta_{k}+V$ for any cusp metric. The constants $C_{1}^{\prime}, C_{2}^{\prime}$ are still given by (3.4), 3.5) since in [32, Proposition 14] only the principal symbol plays a rôle for $p \geq 1 / n$. The coefficient $C_{3}^{\prime}$ can be computed if we assume that the metric is 
exact. It will depend on the zeta function of $\Delta^{M}+V_{0}$. The computation is similar to (3.7).

Concerning essential self-adjointness, the hypothesis on the regularity of the potential part can be weakened using 7] for elliptic operators of order 2 . In the result on the absence of the essential spectrum, one can replace $V$ by $W \in L_{\text {loc }}^{\infty}$, where $V-W$ tends to 0 as $x$ tends to infinity using the Rellich-Kondrakov lemma and the ellipticity of $H_{0}$.

\section{The AnAlysis of the ESSEntial SPECTRUM}

In this section we prove Theorem [1.3, part (11); see Proposition [5.2, We also diagonalize the Laplacian in two different ways. The first one, carried out in Section 5.1, exhibits some key invariant subspaces. It also allows us to compute the essential spectrum. The second one, given in Section 6.1, goes one step beyond and reformulates the problem in some "Euclidean" variables. This will be fully used for the positive commutator techniques; see Section 6 .

We fix $k \in\{0, \ldots, n\}$ and $p>0$ and introduce the constants

$$
c_{0}:=((2 k+2-n) p-1) / 2, \quad c_{1}:=((2 k-2-n) p+1) / 2 .
$$

The set of thresholds is defined as follows:

$$
\begin{aligned}
& \text { for } p<1, \kappa(p)= \begin{cases}\emptyset, & \text { if } b_{k}(M)=b_{k-1}(M)=0, \\
\{0\}, & \text { otherwise; }\end{cases} \\
& \text { for } p=1, \kappa(p)=\left\{c_{i}^{2} \in\left\{c_{0}^{2}, c_{1}^{2}\right\} ; b_{k-i}(M) \neq 0\right\} ; \\
& \text { for } p>1, \kappa(p)=\emptyset .
\end{aligned}
$$

5.1. The high and low energy forms decomposition. We proceed as in [16] and we restrict to metrics which near $M=\{x=0\}$ are of the form

$$
g_{p}^{\text {model }}=x^{2 p}\left(\frac{d x^{2}}{x^{4}}+h\right) .
$$

Here $h$ is independent of $x$. We fix $k$ and localize our computation to the end $X^{\prime}:=(0, \varepsilon) \times M \subset X$. The objects we study do not depend on $\varepsilon$. We introduce

$$
\mathscr{K}:=L^{2}\left((0, \varepsilon), x^{(n-2 k) p-2} d x\right) .
$$

Using (3.2), we get

$$
L^{2}\left(X^{\prime}, \Lambda^{k} X\right)=\mathscr{K} \otimes\left(L^{2}\left(M, \Lambda^{k} M\right) \oplus \frac{d x}{x^{2}} \wedge L^{2}\left(M, \Lambda^{k-1} M\right)\right) .
$$

Setting $\mathscr{H}_{0}:=\mathscr{K} \otimes \operatorname{ker}\left(\Delta_{k}^{M}\right)$ and $\mathscr{H}_{1}:=\mathscr{K} \otimes \operatorname{ker}\left(\Delta_{k-1}^{M}\right)$ and with a slight abuse of notation, this gives

$$
L^{2}\left(X^{\prime}, \Lambda^{k} X\right)=\mathscr{H}_{1} \oplus \mathscr{H}_{\mathrm{h}}=\mathscr{H}_{1_{0}} \oplus \mathscr{H}_{1_{1}} \oplus \mathscr{H}_{\mathrm{h}},
$$

where the space of high energy forms $\mathscr{H}_{\mathrm{h}}$ is by definition the orthogonal complement of $\mathscr{H}_{1}:=\mathscr{H}_{0} \oplus \mathscr{H}_{1_{1}}$. This terminology is justified by the next proposition. See also [26] for a similar phenomenon with a different proof, and 23, 35] for related applications of pseudodifferential operators in determining essential spectra of Laplacians. 
Proposition 5.1. The Laplacian $\Delta_{k}$ on $X^{\prime}$ stabilizes the decomposition (5.5). Let $\Delta_{k}^{\mathrm{l}_{0}}, \Delta_{k}^{\mathrm{l}_{1}}$ and $\Delta_{k}^{\mathrm{h}}$ be the Friedrichs extensions of the restrictions of $\Delta_{k}$ to these spaces, respectively. Then $\Delta_{k}^{\mathrm{h}}$ has compact resolvent, and

$$
\Delta_{k}^{\mathrm{l}_{0}}=\left(D^{*} D+c_{0}^{2} x^{2-2 p}\right) \otimes 1, \quad \Delta_{k}^{\mathrm{l}_{1}}=\left(D^{*} D+c_{1}^{2} x^{2-2 p}\right) \otimes 1,
$$

where $c_{0}, c_{1}$ are defined by (5.1) and $D$ is the closure of $x^{2-p} \partial_{x}-c_{0} x^{1-p}$ with initial domain $\mathcal{C}_{c}^{\infty}((0, \varepsilon))$ in $\mathscr{K}$.

Proof. The de Rham operator on $X^{\prime}$ stabilizes the orthogonal decomposition (5.5), so the Laplacian $d \delta+\delta d$ does the same. Let $P$ denote the orthogonal projection in $L^{2}\left(M, \Lambda^{k} M \oplus \Lambda^{k-1} M\right)$ onto the finite-dimensional space $\operatorname{ker}\left(\Delta_{k}^{M}\right) \oplus \operatorname{ker}\left(\Delta_{k-1}^{M}\right)$ of harmonic forms. Choose a real Schwartz cut-off function $\psi \in \mathcal{S}(\mathbb{R})$ with $\psi(0)=1$. Then $\psi(\xi) P$ defines a suspended operator of order $-\infty$ (see, e.g., [31, Section 2]). From (3.6) we see that $\mathcal{N}\left(x^{2 p} \Delta_{k}\right)(\xi)+\psi^{2}(\xi) P$ is strictly positive, hence invertible for all $\xi \in \mathbb{R}$. By the surjectivity of the normal operator (which is essentially the statement that the restriction map from $\mathcal{C}^{\infty}(X)$ to $\mathcal{C}^{\infty}(M)$ is surjective; see 30, Eq. (12)]), there exists $R \in \Psi_{c}^{-\infty}\left(X, \Lambda^{k} X\right)$ such that in the decomposition (3.2) over $M, \mathcal{N}(R)(\xi)=\psi(\xi) P$. Fix $\phi \in \mathcal{C}_{c}^{\infty}(X)$ which equals 1 on the complement of $X^{\prime}$ in $X$, and yet another cut-off function $\eta$ on $\bar{X}$ which is 1 near $M$ and such that $\eta \phi=0$. By multiplying $R$ both to the left and to the right by $\eta$ we can assume that $R \phi=\phi R=0$, without changing $\mathcal{N}(R)$. The Schwartz kernel of $R$ can be chosen explicitly:

$$
\kappa_{R}\left(x, x^{\prime}, z, z^{\prime}\right)=\eta(x) \hat{\psi}\left(\frac{x-x^{\prime}}{x^{2}}\right) \eta\left(x^{\prime}\right) \kappa_{P}\left(z, z^{\prime}\right),
$$

where $\kappa_{P}$ is the Schwartz kernel of $P$ on $M^{2}$ and $\hat{\psi}$ is the Fourier transform of $\psi$. Assume now that $\hat{\psi}$ has compact support; thus $R$ preserves the space $\mathcal{C}_{c}^{\infty}\left(X^{\prime}, \Lambda^{k} X\right)$. Let $R_{p}:=x^{-p} R \in x^{-p} \Psi_{c}^{-\infty}\left(X, \Lambda^{k} X\right)$. Then $R_{p}^{*} R_{p} \in x^{-2 p} \Psi_{c}^{-\infty}\left(X, \Lambda^{k} X\right)$ is symmetric on $\mathcal{C}_{c}^{\infty}\left(X, \Lambda^{k} X\right)$ with respect to $d g_{p}^{\text {model }}$. Moreover $\Delta_{k}+R_{p}^{*} R_{p}$ is fully elliptic, so by [32, Theorem 17], it is essentially self-adjoint on $\mathcal{C}_{c}^{\infty}\left(X, \Lambda^{k} X\right)$ and has purely discrete spectrum. Now, noticing that $R$ preserves the decomposition (5.5), and acts by 0 on $\mathscr{H}_{\mathrm{h}}$ and by using the decomposition principle [16, Proposition C.3] for $\Delta_{k}$ and $\Delta_{k}+R_{p}^{*} R_{p}$, we deduce that $\sigma_{\mathrm{ess}}\left(\Delta_{k}+R_{p}^{*} R_{p}\right)=\sigma_{\mathrm{ess}}\left(\Delta_{k}^{\mathrm{h}}\right)=\emptyset$.

For the low energy forms, one gets $\Delta_{k}^{\mathrm{l}_{0}}=-x^{(2 k-n) p} x^{2} \partial_{x} x^{(n-2 k-2) p} x^{2} \partial_{x} \otimes 1$, acting in $\mathscr{H}_{1_{0}}$, and $\Delta_{k}^{\mathrm{l}_{1}}=-x^{2} \partial_{x} x^{(2 k-2-n) p} x^{2} \partial_{x} x^{(n-2 k) p} \otimes 1$ acting in $\mathscr{H}_{1_{1}}$. The proof is finished by expanding $D^{*} D$.

Proposition 5.2. Let $\left(X, g_{p}^{\prime}\right)$ be a Riemannian manifold with metric $g_{p}^{\prime}$ satisfying the bounds (A.1) and (A.2) with respect to $g_{p}=x^{2 p} g_{0}$, for some exact cusp metric $g_{0}$.

(1) For $0<p \leq 1$, consider the Friedrichs extension of $\Delta_{k}$. Then its essential spectrum is given by $[\inf (\kappa(p)), \infty)$.

(2) If $p>1$ and $g_{p}^{\prime}:=g_{p}^{\text {model }}$ is the unperturbed metric given in (5.3), then every self-adjoint extension of $\Delta_{k}$ has empty essential spectrum.

In particular, when $X$ is complete (i.e., $p \leq 1$ ) the Laplacian of $g_{p}$ on forms of degree 0 and 1 always has non-empty essential spectrum. If moreover the boundary at infinity $M$ has at least one orientable connected component, then the same holds for forms of degrees $n-1$ and $n$. Note that Theorem 1.2 does not follow from Proposition 5.2 since in Section 2 we do not assume the metric to be exact. 
Proof. We start with the complete case. For a smooth complete metric, the essential self-adjointness is a well-known general fact [11. As the metric we consider is not necessarily smooth, we consider the Friedrichs extension. In the exact case, $g_{p}$ is asymptotically isometric to the model metric (5.3) in the sense of (A.1). Using Proposition A.1, in order to compute the essential spectrum we may replace $h(x)$ in (2.1) by the metric $h_{0}:=h(0)$ on $M$, extended to a symmetric 2-tensor constant in $x$ near $M$, and we may also set $\rho=0$. By [16, Lemma C.1], the essential spectrum $\sigma_{\text {ess }}\left(\Delta_{k}\right)$ is given by $\bigcup_{i} \sigma_{\text {ess }}\left(\Delta_{k}^{l_{i}}\right)$ on $X^{\prime}$ of Proposition 5.1. To conclude, remark that the essential spectrum of $D^{*} D$ is $[0, \infty)$ and the essential spectrum of $\Delta_{k}^{\mathrm{I}_{i}}$ is $\left[\lim _{x \rightarrow 0} c_{i} x^{2-2 p}, \infty\right)$ since a bounded potential tending to 0 is a relatively compact perturbation and thus does not affect the essential spectrum. One may also compute the essential spectrum using (6.3).

Now let $p>1$. The metric is no longer complete, so one cannot apply Proposition A.1. By [16, Lemma B.1] and by the Krein formula, all self-adjoint extensions have the same essential spectrum, so it is enough to consider the Friedrichs extension of $\Delta_{k}$. We now use Proposition 5.1 and [16, Lemma C.1]. The operator $D^{*} D$ is nonnegative, so the spectrum of $\Delta_{k}^{1_{i}}$ is contained in $\left[\varepsilon^{2-2 p} c_{i}^{2}, \infty\right)$. By [16, Lemma C.1], the essential spectrum does not depend on the choice of $\varepsilon$. Now we remark that $p>1$ implies $\lim _{\varepsilon \rightarrow 0} \varepsilon^{2-2 p}=\infty$ and also that $c_{i} \neq 0, i=0,1$ for the constants $c_{0}, c_{1}$ defined by (5.1). Indeed, the equality $c_{i}=0$ would imply $1 / p= \pm(2 k \pm 2-n) \in \mathbb{Z}$, which contradicts $p>1$. Thus by letting $\varepsilon \rightarrow 0$ we conclude that the essential spectrum of $\Delta_{k}$ is empty.

\section{The Mourre estimate}

In Mourre theory, one has to construct a conjugate operator in order to obtain the positivity of a commutator. To this purpose in Section 6.1 we write $\Delta_{k}$ near infinity in terms of the function $L$ defined in equation (1.4) instead of the boundary defining function $x$. In Section 6.2 we construct the conjugate operator in terms of this new variable. Its support is in the cusp, so one can restrict the analysis there. In Section 6.3 we prove the Mourre estimate for the unperturbed metric in Theorem 6.2. Finally, in Section 6.4 we show the main Theorem 1.3 for conformal perturbations of the model metric (5.3). In this section, we concentrate on the complete case, i.e. $p \leq 1$; otherwise, there is no essential spectrum and the whole analysis becomes trivial (one may take the conjugate operator to be 0 ).

6.1. Diagonalization of the free Laplacian. We now construct a partial isometry. We carry out the analysis on the cusp $X^{\prime}$. We start with (5.5) and work on $\mathscr{K}$; see (5.4). We conjugate first through the unitary transformation

$$
L^{2}\left(x^{(n-2 k) p-2} d x\right) \rightarrow L^{2}\left(x^{p-2} d x\right), \quad \phi \mapsto x^{(n-2 k-1) p / 2} \phi .
$$

Then we proceed with the change of variables

$$
r:=L(1 / x), \quad \text { where } L \text { is given by (1.4). }
$$

Therefore, $\mathscr{K}$ is unitarily sent into $\mathscr{K}_{0}:=L^{2}((c, \infty), d r)$ for some positive $c$. By tensoring with the identity on $M$, we have constructed a unitary transformation:

$$
\mathcal{U}: L^{2}\left((0, \varepsilon) \times M, \Lambda^{k} X, g_{p}^{\text {model }}\right) \longrightarrow L^{2}\left((c, \infty) \times M, \Lambda^{k} X, d r^{2}+h\right)=: \mathscr{H}_{0} .
$$

We set $X_{0}^{\prime}:=(c, \infty) \times M$. Note that $\mathcal{U}$ is an isomorphism between $\mathcal{C}_{c}^{\infty}\left(X^{\prime}, \Lambda^{*} X\right)$ and $\mathcal{C}_{c}^{\infty}\left(X_{0}^{\prime}, \Lambda^{*} X\right)$. 
Set $L_{0}:=\mathcal{U} L \mathcal{U}^{-1}$, where $L$ is the operator of multiplication by the function $L$ given in (1.4). We choose $\varepsilon$ small enough so that $L_{0}$ is the operator of multiplication by $(r, m) \mapsto r$ in $\mathscr{H}_{0}$. We consider the closure of $\Delta_{k, 0}:=\mathcal{U} \Delta_{k} \mathcal{U}^{-1}$ defined above in $\mathcal{C}_{c}^{\infty}\left(X_{0}^{\prime}, \Lambda^{*} X\right)$. Recalling (5.5), it acts on $\mathcal{C}_{c}^{\infty}((c, \infty)) \otimes \mathcal{C}^{\infty}\left(M, \Lambda^{k} M \oplus \Lambda^{k-1} M\right)$ as

$$
\Delta_{k, 0}=\sum_{i=0,1}\left(-\partial_{r}^{2}+V_{p_{i}}\right) \otimes P_{\operatorname{ker}\left(\Delta_{k-i}^{M}\right)}+\Delta_{k, 0}^{\mathrm{h}} \otimes P_{0}^{\perp}, \quad V_{p_{i}}(r)= \begin{cases}c_{i}^{2} & \text { for } p=1 \\ a_{i} / r^{2} & \text { for } p<1\end{cases}
$$

for certain $a_{i}$, where $P_{0}:=P_{\operatorname{ker}\left(\Delta_{k}^{M}\right)} \oplus P_{\operatorname{ker}\left(\Delta_{k-1}^{M}\right)}$, and $c_{0}, c_{1}$ are defined in (5.1). Note that $\Delta_{k, 0}$ is not self-adjoint. Its spectrum is $\mathbb{C}$. We will only use it as an auxiliary operator.

6.2. The conjugate operator. We now construct a conjugate operator so as to establish a Mourre estimate for the Laplacian acting on $k$-forms for the free metric $g=g_{p}^{\text {model }}$, given by (5.3). This section is close to [16, Section 5.3] for the commutator properties, but the proof of the Mourre estimate differs, so we provide full details. Let $\xi \in \mathcal{C}^{\infty}(c, \infty)$ such that the support of $\xi$ is contained in $[3 c, \infty)$ and such that $\xi(r)=r$ for $r \geq 4 c$ and let $\tilde{\chi} \in \mathcal{C}^{\infty}(c, \infty)$ with support in $[2 c, \infty)$, which equals 1 on $[3 c, \infty)$. By abuse of notation, we denote $\tilde{\chi} \otimes 1$ and $\xi \otimes 1$ in $\mathcal{C}^{\infty}\left(X_{0}^{\prime}\right)$ by $\tilde{\chi}$ and $\xi$, respectively. We also write with the same symbol a function and the operator of multiplication by that function acting in a Hilbert space. Choose $\Phi \in \mathcal{C}_{c}^{\infty}(\mathbb{R})$ with $\Phi(x)=x$ on $[-1,1]$, and set $\Phi_{\Upsilon}(x):=\Upsilon \Phi(x / \Upsilon)$. We define on $\mathcal{C}_{c}^{\infty}\left(X_{0}^{\prime}, \Lambda^{k} X\right)$ a micro-localized version of the generator of dilations:

$$
S_{\Upsilon, 0}:=\tilde{\chi}\left(\Phi_{\Upsilon}\left(-i \partial_{r}\right) \xi+\xi \Phi_{\Upsilon}\left(-i \partial_{r}\right)\right) \otimes P_{0} \tilde{\chi}
$$

The operator $\Phi_{\Upsilon}\left(-i \partial_{r}\right)$ is defined on the real line by $\mathscr{F}^{-1} \Phi_{\Upsilon}(\cdot) \mathscr{F}$, where $\mathscr{F}$ is the unitary Fourier transform. We also denote the closure of $S_{\Upsilon, 0}$ by the same symbol. Choose $\widetilde{\mu}_{0} \in \mathcal{C}^{\infty}\left(X_{0}^{\prime}\right)$ such that $\left.\widetilde{\mu}_{0}\right|_{[2 c, \infty) \times M}=1$, and extend it by 0 above the compact part of $X$. Set the operator of multiplication $\widetilde{\mu}:=\mathcal{U}^{-1} \widetilde{\mu}_{0} \mathcal{U}$. On $\mathcal{C}_{c}^{\infty}\left(X, \Lambda^{k} X\right)$, we set

$$
S_{\Upsilon}:=\mathcal{U}^{-1} S_{\Upsilon, 0} \mathcal{U} \widetilde{\mu}
$$

and denote also by $S_{\Upsilon}$ its closure. Note that $S_{\Upsilon}$ does not stabilize $\mathcal{C}_{c}^{\infty}\left(X, \Lambda^{k} X\right)$ since $\Phi_{\Upsilon}\left(-i \partial_{r}\right)$ acts as a convolution with a function with non-compact support. However, $\mathcal{C}_{c}^{\infty}\left(X, \Lambda^{k} X\right)$ is sent into the restriction of the Schwartz space $\mathcal{U}^{-1}(\tilde{\chi} \mathscr{S}(\mathbb{R}) \otimes$ $\left.\operatorname{Im}\left(P_{0}\right)\right)$.

When $\Upsilon=\infty$, this operator is also self-adjoint and one recovers the conjugate operator initiated in [10] for the case of the Laplacian. The drawback of this operator is that it does not allow very singular perturbation theory such as the one of the metric we consider; see also [16. Since here $\Phi_{\Upsilon}$ is with compact support, one is able to replace $S_{\Upsilon}$ by $L$ in the theory of perturbation. We prove some results of compatibility in the next lemma. From now on, $\Upsilon$ is finite.

Lemma 6.1. For all $\Upsilon \geq 1$, the operator $S_{\Upsilon}$ has the following properties:

(1) it is essentially self-adjoint on $\mathcal{C}_{c}^{\infty}\left(X, \Lambda^{k} X\right)$;

(2) for every $s, t \in \mathbb{R}^{+}, L^{-2} S_{\Upsilon}^{2} \mathcal{D}\left(\Delta_{k}^{s}\right) \subset \mathcal{D}\left(\Delta_{k}^{t}\right)$;

(3) for all $s \in[0,2], \mathcal{D}\left(L^{s}\right) \subset \mathcal{D}\left(\left|S_{\Upsilon}\right|^{s}\right)$. 
Proof. We compare $S_{\Upsilon}$ with the operator $L$, which is essentially self-adjoint on $\mathcal{C}_{c}^{\infty}\left(X, \Lambda^{k} X\right)$. Moreover $L$ stabilizes the decomposition (5.5), and we denote by $L_{1}$ its restriction to $\mathscr{H}_{1}=\mathscr{H}_{1_{0}} \oplus \mathscr{H}_{1_{1}}$. Set $L_{1,0}:=\mathcal{U} L_{1} \mathcal{U}^{-1}$, which is simply multiplication by $r$. For brevity, we write $\Phi_{\Upsilon}$ instead of $\Phi_{\Upsilon}\left(-i \partial_{r}\right)$. Take $\varphi \in \mathcal{C}_{c}^{\infty}\left(X, \Lambda^{k} X\right)$. Note that $\tilde{\varphi}:=\mathcal{U} \tilde{\mu} \varphi \in \mathcal{C}_{c}^{\infty}\left(X_{0}^{\prime}, \Lambda^{k} X\right)$. We have

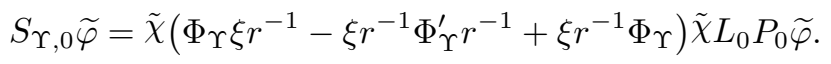

Since $\xi r^{-1}$ is bounded in $L^{2}\left(X_{0}, \Lambda^{k} X\right)$, we get $\left\|S_{\Upsilon, 0} \widetilde{\varphi}\right\| \leq a\left\|L_{0} \widetilde{\varphi}\right\|$, in $L^{2}\left(X_{0}^{\prime}, \Lambda^{k} X\right)$. Remembering $L \geq 1$, we derive that there exists $a^{\prime}$ so that $\left\|S_{\Upsilon \varphi}\right\| \leq a^{\prime}\|L \varphi\|$ in $L^{2}\left(X, \Lambda^{k} X\right)$. To be precise, one needs first to approximate $\widetilde{\varphi}$ with $\widetilde{\varphi}_{\mathbb{R}} \otimes\left(\widetilde{\varphi}_{M}+d r \wedge\right.$ $\left.\widetilde{\varphi}_{M}^{\prime}\right)$, where $\widetilde{\varphi}_{\mathbb{R}} \in \mathcal{C}_{c}^{\infty}\left(X_{0}^{\prime}\right), \widetilde{\varphi}_{M} \in \mathcal{C}^{\infty}\left(M, \Lambda^{k} M\right)$ and $\widetilde{\varphi}_{M}^{\prime} \in \mathcal{C}^{\infty}\left(M, \Lambda^{k-1} M\right)$.

On the other hand, we compute against $\tilde{\varphi}$ in the form sense and get

$$
\begin{aligned}
{\left[S_{\Upsilon, 0}, L_{0}\right]=} & \tilde{\chi}\left(\left[\Phi_{\Upsilon}, r\right] \xi \otimes P_{0}+\xi\left[\Phi_{\Upsilon}, r\right] \otimes P_{0}\right) \tilde{\chi} \\
= & i \tilde{\chi}\left(\Phi_{\Upsilon}^{\prime} r r^{-1} \xi \otimes P_{0}+\xi r^{-1} r \Phi_{\Upsilon}^{\prime} \otimes P_{0}\right) \tilde{\chi} \\
= & i \tilde{\chi}\left(r^{1 / 2} \Phi_{\Upsilon}^{\prime} r^{1 / 2} r^{-1} \xi \otimes P_{0}+r^{-1} \xi r^{1 / 2} \Phi_{\Upsilon}^{\prime} r^{1 / 2} \otimes P_{0}\right) \tilde{\chi} \\
& +i \tilde{\chi}\left(\left[\Phi_{\Upsilon}^{\prime}, r^{1 / 2}\right] r^{1 / 2} r^{-1} \xi \otimes P_{0}+r^{-1} \xi\left[\Phi_{\Upsilon}^{\prime}, r^{1 / 2}\right] r^{1 / 2} \otimes P_{0}\right) \tilde{\chi}
\end{aligned}
$$

This gives $\left|\left\langle S_{\Upsilon, 0} \widetilde{\varphi}, L_{0} \widetilde{\varphi}\right\rangle-\left\langle L_{0} \widetilde{\varphi}, S_{\Upsilon, 0} \widetilde{\varphi}\right\rangle\right| \leq b\left\|L_{0}^{1 / 2} \widetilde{\varphi}\right\|^{2}$ in $L^{2}\left(X_{0}^{\prime}, \Lambda^{k} X\right)$. Since $L \geq 1$,

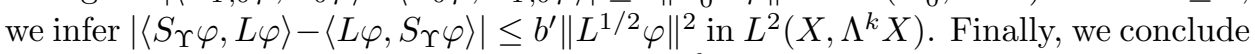
that $S_{\Upsilon}$ is essentially self-adjoint on $\mathcal{C}_{c}^{\infty}\left(X, \Lambda^{k} X\right)$ by using [38, Theorem X.37].

We turn to point (2). By interpolation, it is enough to show that $L^{-2} S_{\Upsilon}^{2}\left(\Delta_{k}+1\right)^{s}$ and $S_{\Upsilon}^{2} L^{-2}\left(\Delta_{k}+1\right)^{s}$, defined on $\mathcal{C}_{c}^{\infty}\left(X, \Lambda^{k} X\right)$, extend to bounded operators in $L^{2}\left(X, \Lambda^{k} X\right)$, for all $s \in \mathbb{N}$. The treatment being similar, we deal only with the first term.

Since $\tilde{\chi}$ stabilizes all the Sobolev spaces in $\mathbb{R}$, using (6.3) we obtain that there is $c$ so that

$$
\left\|\left(\Delta_{\mathbb{R}}+i\right)^{-s} \otimes P_{0} \tilde{\chi}\left(\Delta_{k, 0}+i\right)^{s} \widetilde{\varphi}\right\| \leq c\|\widetilde{\varphi}\|,
$$

for all $\widetilde{\varphi}$ taken as above. Here $\Delta_{\mathbb{R}}$ denotes the positive Laplacian in $L^{2}(\mathbb{R})$. Now remark that thanks to the cut-off functions $\tilde{\chi}$, there is $g \in \mathcal{C}_{c}^{\infty}(\mathbb{R})$ such that

$$
S_{\Upsilon, 0}=\tilde{\chi}\left(2 r \Phi_{\Upsilon}+i \Phi_{\Upsilon}^{\prime}+g \Phi_{\Upsilon}+\Phi_{\Upsilon} g\right) \otimes P_{0} \tilde{\chi} .
$$

Then thanks to (6.6) and by going back to $\varphi$ as above, the result follows if

$$
r^{-2}\left(2 r \Phi_{\Upsilon}+i \Phi_{\Upsilon}^{\prime}+g \Phi_{\Upsilon}+\Phi_{\Upsilon} g\right) \tilde{\chi}^{2}\left(2 r \Phi_{\Upsilon}+i \Phi_{\Upsilon}^{\prime}+g \Phi_{\Upsilon}+\Phi_{\Upsilon} g\right)\left(\Delta_{\mathbb{R}}+1\right)^{s}
$$

defined on $\mathcal{C}_{c}^{\infty}(\mathbb{R})$ extends to a bounded operator. This follows easily by commuting all the $r$ to the left and by using the stability of Sobolev spaces under the multiplication by a smooth function with bounded derivatives.

We now focus on point (3). Using (6.8), we have shown in particular that $S_{\Upsilon}^{2} L^{-2}$ is bounded in $L^{2}\left(X, \Lambda^{k} X\right)$. We infer $\left\|S_{\Upsilon}^{2} \varphi\right\|^{2} \leq c\left\|L^{2} \varphi\right\|$ for all $\varphi \in \mathcal{C}_{c}^{\infty}\left(X, \Lambda^{k} X\right)$. Taking a Cauchy sequence, we deduce $\mathcal{D}\left(L^{2}\right) \subset \mathcal{D}\left(S_{\Upsilon}^{2}\right)$. An argument of interpolation concludes the proof.

6.3. The Mourre estimate for the unperturbed metric. The main result of this section is the following Mourre estimate for the free operator $\Delta_{k}$. The proof is more involved than in [16] although the computations of commutators are essentially the same. The problem comes from the fact that $\Delta_{k}$ has two thresholds, not 
just one like the magnetic Laplacian acting on functions. Above the two thresholds we can pursue the same analysis as [16]. Morally speaking, as in 3-body problems, when we localize the energy between the thresholds, the smallest one will yield the positivity of the commutator, and the largest one will bring more compactness. The technical problem comes from the fact that the resolvent of $\Delta_{k}$, and therefore the spectral measure, does not stabilize the decomposition (5.5). To have such a decomposition, one would have to uncouple the cusp part from the compact part. Here some caution should be exercised, since considering the Friedrichs extension of $\Delta_{k}$, on the cusp and on the compact part, would be too singular a perturbation, even though it is enough for the study of the essential spectrum; see 16, Lemma C.1]. We uncouple only the low energy part.

We recall the regularity classes of the Mourre theory. We refer to [1] for a more thorough discussion of these matters. Take $H$ and $A$ to be two self-adjoint operators acting in a Hilbert space $\mathscr{H}$. We say that $H \in \mathcal{C}^{k}(A)$ if $t \mapsto e^{-i t A}(H+i)^{-1} e^{i t A}$ is strongly $\mathcal{C}^{k}$ in $\mathcal{B}(\mathscr{H})$. Moreover, if $e^{i t A} \mathcal{D}(H) \subset \mathcal{D}(H)$, this is equivalent to the fact that $H \in \mathcal{C}^{k}\left(A ; \mathcal{D}(H), \mathcal{D}(H)^{*}\right)$; i.e., the function $t \mapsto e^{-i t A} H e^{i t A}$ is strongly $\mathcal{C}^{k}$ in $\mathcal{B}\left(\mathcal{D}(H), \mathcal{D}(H)^{*}\right)$. Recall that we use the Riesz isomorphism to identify $\mathscr{H}$ with $\mathscr{H}^{*}$. We now give the main result of this section.

Theorem 6.2. For every $\Upsilon \geq 1$ and $p \leq 1$ we have $e^{i t S_{\Upsilon}} \mathcal{D}\left(\Delta_{k}\right) \subset \mathcal{D}\left(\Delta_{k}\right)$ and $\Delta_{k} \in \mathcal{C}^{2}\left(S_{\Upsilon} ; \mathcal{D}\left(\Delta_{k}\right), \mathscr{H}\right)$. Given an interval $\mathcal{J}$ which does not intersect $\kappa(p)$ (see (5.2) ), let $c_{\mathcal{J}}<d(\inf (\mathcal{J}),\{c \in \kappa(p), c \leq \inf (\mathcal{J})\})$. Then there exist $\varepsilon_{\Upsilon}>0$ and a compact operator $K_{\Upsilon}$ such that the inequality

$$
E_{\mathcal{J}}\left(\Delta_{k}\right)\left[\Delta_{k}, i S_{\Upsilon}\right] E_{\mathcal{J}}\left(\Delta_{k}\right) \geq\left(4 c_{\mathcal{J}}-\varepsilon_{\Upsilon}\right) E_{\mathcal{J}}\left(\Delta_{k}\right)+K_{\Upsilon}
$$

holds in the sense of forms, and such that $\varepsilon_{\Upsilon}$ tends to 0 as $\Upsilon$ goes to infinity.

We now establish a series of lemmata and prove this theorem at the end of the section.

Lemma 6.3. The commutators $\left[\Delta_{k}, i S_{\Upsilon}\right]$ and $\left[\left[\Delta_{k}, i S_{\Upsilon}\right], i S_{\Upsilon}\right]$, taken in the form sense on $\mathcal{C}_{c}^{\infty}\left(X, \Lambda^{k} X\right)$, extend to bounded operators in $\mathscr{H}$.

Proof. Let $\varphi \in \mathcal{C}_{c}^{\infty}\left(X, \Lambda^{k} X\right)$. Set $\widetilde{\varphi}:=\mathcal{U} \tilde{\mu} \varphi \in \mathcal{C}_{c}^{\infty}\left(X_{0}^{\prime}, \Lambda^{k} X\right)$ and write $\Phi_{\Upsilon}$ instead of $\Phi_{\Upsilon}\left(-i \partial_{r}\right)$. To justify the computations in the tensor product form in $L^{2}\left(X_{0}^{\prime}, \Lambda^{k} X\right)$ we approximate $\widetilde{\varphi}$ as in Lemma 6.1.

For instance, for the commutator $\left[\Delta_{k}, S_{\Upsilon}\right]$, we use the transformation $\mathcal{U}$. We show that $\left|\left\langle\widetilde{\varphi},\left[\Delta_{k, 0}, S_{\Upsilon, 0}\right] \widetilde{\varphi}\right\rangle\right| \leq c\|\widetilde{\varphi}\|^{2}$. Thanks to the support of the commutator, we can remove $\tilde{\mu}$ and infer $\left|\left\langle\varphi,\left[\Delta_{k}, S_{\Upsilon}\right] \varphi\right\rangle\right| \leq c^{\prime}\|\varphi\|^{2}$. To carry out the computation, we use the expression obtained in (6.3). The high energy part plays no rôle. We drop $P_{0}$ for convenience, and it is enough to compute in the form sense in $\mathcal{C}_{c}^{\infty}(\mathbb{R})$.

We show something stronger. We first deal with the part in $\partial_{r}$. We now rewrite $S_{\Upsilon, 0}$ as in (6.7). By commuting till all the $\partial_{r}$ are next to a $\Phi_{\Upsilon}$, we get

$$
\left[\partial_{r}^{2}, \tilde{\chi}\left(\Phi_{\Upsilon} \xi+\xi \Phi_{\Upsilon}\right) \tilde{\chi}\right]=4 \tilde{\chi} \partial_{r} \Phi_{\Upsilon} \tilde{\chi}+L_{0}^{-1} \times \text { bounded }
$$


Here $\times$ means the composition of two operators. For simplicity, we treat only the part

$$
\begin{aligned}
\tilde{\mu}_{0} \partial_{r}\left[\partial_{r}, \tilde{\chi} \Phi_{\Upsilon} \xi \tilde{\chi}\right] \tilde{\mu}_{0}=\tilde{\chi}^{\prime} \partial_{r} \Phi_{\Upsilon} \xi \tilde{\chi}+\tilde{\chi}^{\prime \prime} \Phi_{\Upsilon} \xi \tilde{\chi}+\tilde{\chi} \partial_{r} \Phi_{\Upsilon} \xi^{\prime} \tilde{\chi}+\tilde{\chi}^{\prime} \Phi_{\Upsilon} \xi^{\prime} \tilde{\chi} \\
+\tilde{\chi} \partial_{r} \Phi_{\Upsilon} \xi \tilde{\chi}^{\prime}+\tilde{\chi}^{\prime} \Phi_{\Upsilon} \xi \tilde{\chi}^{\prime} \\
=\tilde{\chi} \partial_{r} \Phi_{\Upsilon} \tilde{\chi}+\tilde{\chi}(r+i)^{-1}(r+i) \partial_{r} \Phi_{\Upsilon}\left(1-\xi^{\prime}\right) \tilde{\chi}+\tilde{\chi}^{\prime} \partial_{r} \Phi_{\Upsilon}(r+i)(r+i)^{-1} \xi \tilde{\chi} \\
\quad+\tilde{\chi}^{\prime \prime}(r+i)^{-1}(r+i) \Phi_{\Upsilon} \xi^{\prime} \tilde{\chi}+\tilde{\chi}(r+i)^{-1}(r+i) \partial_{r} \Phi_{\Upsilon} \xi \tilde{\chi}^{\prime}+L_{0}^{-1} \times \text { bounded } \\
=\tilde{\chi} \partial_{r} \Phi_{\Upsilon} \tilde{\chi}+L_{0}^{-1} \times \text { bounded, }
\end{aligned}
$$

since $\left[r, \partial_{r} \Phi_{\Upsilon}\right],\left[r, \Phi_{\Upsilon}\right]$ and $(r+i)^{-1} \xi \tilde{\chi}$ are bounded and $1-\xi^{\prime}$ and $\chi^{\prime}$ are in $\mathcal{C}_{c}^{\infty}$. In the same way, we obtain $\left[\left[\partial_{r}^{2}, \tilde{\chi}\left(\Phi_{\Upsilon} \xi+\xi \Phi_{\Upsilon}\right) \tilde{\chi}\right], \tilde{\chi}\left(\Phi_{\Upsilon} \xi+\xi \Phi_{\Upsilon}\right) \tilde{\chi}\right]=8 \tilde{\chi}^{2} \partial_{r} \Phi_{\Upsilon} \tilde{\chi}^{2}+$ bounded.

For $p<1$, the potential part $V_{p_{i}}$ arises. We extend it smoothly in $\mathbb{R}$ and with compact support in $\mathbb{R}^{-}$. We treat its first commutator:

$$
\begin{aligned}
{\left[V_{p_{i}}, \tilde{\chi}\left(\Phi_{\Upsilon} \xi+\xi \Phi_{\Upsilon}\right) \tilde{\chi}\right]=} & \tilde{\chi} V \Phi_{\Upsilon}(r+i)(r+i)^{-1} \xi \tilde{\chi}-\tilde{\chi}(r+i)^{-1}(r+i) \Phi_{\Upsilon} V \xi \tilde{\chi} \\
& +\tilde{\chi} \xi V \Phi_{\Upsilon} \tilde{\chi}-\chi\left(r^{2}+1\right)^{-1} \xi\left(r^{2}+1\right) \Phi_{\Upsilon} V \xi \tilde{\chi} \\
= & L_{0}^{-1} \times \text { bounded, }
\end{aligned}
$$

since $\left[r, \Phi_{\Upsilon}\right]$ and $\left[r^{2}, \Phi_{\Upsilon}\right](r+i)^{-1}$ are bounded.

Consider finally the second commutator of $V_{p_{i}}$. We treat only the most singular part $\left[\left[V_{p_{i}}, \Phi_{\Upsilon}\right] r, \Phi_{\Upsilon}^{\prime} r\right]=\left[\left[V_{p_{i}}, \Phi_{\Upsilon}\right], \Phi_{\Upsilon}\right] r^{2}+i \Phi_{\Upsilon}\left[\Phi_{\Upsilon}^{\prime}, V_{p_{i}}\right] r+\left[V_{p_{i}}, \Phi_{\Upsilon}\right] \Phi_{\Upsilon}^{\prime \prime}-$ $i\left[V_{p_{i}}, \Phi_{\Upsilon}\right] r \Phi_{\Upsilon}^{\prime}$. As above, commute till $r$ touches $V_{p_{i}}$ so as to get the boundedness.

As pointed out in [13, the $\mathcal{C}^{1}$ assumption of regularity is essential in the Mourre theory and should be checked carefully. See 20] for a different approach.

Lemma 6.4. For $\Upsilon \geq 1$, one has $\Delta_{k} \in \mathcal{C}^{1}\left(S_{\Upsilon}\right)$ and $e^{i t S_{\Upsilon}} \mathcal{D}\left(\Delta_{k}\right) \subset \mathcal{D}\left(\Delta_{k}\right)$.

Proof. We check the hypothesis of Lemma [16, Lemma A.2] so as to get the $\mathcal{C}^{1}$ property. Let $\chi_{n}(r):=\chi(r / n)$ and $\mathscr{D}:=\mathcal{C}_{c}^{\infty}\left(X, \Lambda^{k} X\right)$. Since $\left\|\chi_{n}^{\prime}\right\|_{\infty}$ and $\left\|\chi_{n}^{\prime \prime}\right\|_{\infty}$ are uniformly bounded, we get that $\sup _{n}\left\|\chi_{n}\right\|_{\mathcal{D}(H)}$ is finite. By Lemma 6.1, $\mathscr{D}$ is a core for $S_{\Upsilon}$. Assumption (1) is obvious, assumption (2) holds since $\left(1-\chi_{n}\right)$ has support in $[2 n, \infty)$ and assumption (3) follows from the fact that $H$ is elliptic, so the resolvent of $\Delta_{k}$ sends $\mathscr{D}$ into $\mathcal{C}^{\infty}\left(X, \Lambda^{k} X\right)$. The point (A.6) follows from Lemma 6.3. It remains to show (A.5). Let $\phi \in \mathcal{C}^{\infty}\left(X, \Lambda^{k} X\right) \cap \mathcal{D}\left(\Delta_{k}\right)$ and set $\tilde{\phi}:=\mathcal{U} \tilde{\mu} \phi$. We get $i S_{\Upsilon, 0}\left[\Delta_{k, 0}, \chi_{n}\right] \tilde{\phi}=\tilde{\chi}\left(2 \Phi_{\Upsilon}\left(\partial_{r}\right)+\left[\xi, \Phi_{\Upsilon}\left(\partial_{r}\right)\right] \xi^{-1}\right) \tilde{\chi} P_{0}\left(2 \xi \chi_{n}^{\prime} \partial_{r} \tilde{\phi}+\xi \chi_{n}^{\prime \prime} \tilde{\phi}\right)$. Both terms are tending to 0 because $\operatorname{supp}\left(\chi_{n}^{\prime}\right) \subset[n, 2 n]$ and $\xi \chi_{n}^{(k)}$ tends strongly to 0 on $L^{2}\left(\mathbb{R}^{+}\right)$for $k \geq 1$. Note that one may remove $\widetilde{\mu}$ because of the support. This shows that $\Delta_{k} \in \mathcal{C}^{1}$.

Since $\left[\Delta_{k}, i S_{\Upsilon}\right] \in \mathcal{B}\left(\mathcal{D}\left(\Delta_{k}\right), \mathscr{H}\right)$, [12, Lemma 2] yields the conclusion $e^{i t S_{\Upsilon}} \mathcal{D}\left(\Delta_{k}\right)$ $\operatorname{subset} \mathcal{D}\left(\Delta_{k}\right)$.

Lemma 6.5. Let $\Upsilon \geq 1$. Then $\Delta_{k}$ belongs to $\mathcal{C}^{2}\left(S_{\Upsilon} ; \mathcal{D}\left(\Delta_{k}\right), \mathscr{H}\right)$ for $p \leq 1$.

Proof. From Lemma 6.4, we have the invariance of the domain and from Lemma 6.3. the two commutators of $\Delta_{k}$ with $S_{\Upsilon}$ extend to bounded operators. 
We now introduce an intermediate operator, to be able to deal with the range between the thresholds when both Betti numbers are non-zero. This situation is somewhat similar to adding an anisotropic potential for the Euclidean Laplacian in dimension 1. Let $\dot{\Delta}_{k}$ be the Friedrichs extension of $\Delta_{k}$ over

$$
\mathscr{D}_{1}=\left\{f \in \mathcal{C}_{c}^{\infty}\left(X, \Lambda^{k} X\right): P_{0} f(x, m)=0, \text { for } x=\varepsilon \text { and for all } m \in M\right\}
$$

Here $\varepsilon$ is chosen as in Section 6.1. We note that $\left.\Delta_{k}\right|_{\mathscr{D}_{1}}$ has finite deficiency indices since $P_{0}$ is of finite rank by compactness of $M$. Using the Krein formula and the Stone-Weierstrass theorem, we infer that

$$
\theta\left(\Delta_{k}\right)-\theta\left(\dot{\Delta}_{k}\right) \in \mathcal{K}(\mathscr{H}), \text { for all } \theta \in \mathcal{C}_{c}(\mathbb{R}, \mathbb{C}) .
$$

The main interest of $\dot{\Delta}_{k}$ is that its resolvent stabilizes the low energy part of the decomposition (5.5). Indeed, $\left.\dot{\Delta}_{k}\right|_{\mathscr{H}_{1}}$ is the Dirichlet extension of $\Delta_{k}$ in $\mathscr{H}_{1_{0}}$.

Note that the high energy part interacts with the compact part of $X$.

Remark 6.6. We have $\dot{\Delta}_{k} \in \mathcal{C}^{1}\left(S_{R}\right)$. In fact, because of the support of $\tilde{\chi}, e^{i t S_{\Upsilon, 0}}$ acts as the identity on the orthogonal complement of $L^{2}([c, \infty)) \otimes L^{2}(M)$ in $\mathscr{H}_{0}$. Combining Lemma 6.5 and interpolation, we deduce that $e^{i t S_{\Upsilon}}$ stabilizes the form domain of $\dot{\Delta}_{k}$. Then, repeating Lemma 6.3 and using 6.13), we infer $\dot{\Delta}_{k} \in$ $\mathcal{C}^{1}\left(S_{\Upsilon} ; \mathcal{D}\left(\dot{\Delta}_{k}^{1 / 2}\right), \mathcal{D}\left(\dot{\Delta}_{k}^{1 / 2}\right)^{*}\right)$. Note that $\left[\dot{\Delta}_{k}, i S_{\Upsilon}\right]$ is equal to the operator $\left[\Delta_{k}, i S_{\Upsilon}\right]$ thanks to the support of $S_{\Upsilon}$.

We are now in a position to prove the main result of this section.

Proof of Theorem 6.2. The regularity assumptions follow from Lemmata 6.4 and 6.5. Set $\varphi \in \mathcal{C}_{c}^{\infty}\left(X, \Lambda^{k} X\right)$. Set $\tilde{\varphi}:=\mathcal{U} \tilde{\mu} \varphi$ and write $\Phi_{\Upsilon}$ instead of $\Phi_{\Upsilon}\left(-i \partial_{r}\right)$. To justify the computations in the tensor product form in $L^{2}\left(X_{0}^{\prime}, \Lambda^{k} X\right)$ we approximate $\widetilde{\varphi}$ as in Lemma 6.1. Because of supports, one has $\left\langle\varphi,\left[\Delta_{k}, i S_{\Upsilon}\right] \varphi\right\rangle=$ $\left\langle\widetilde{\varphi},\left[\Delta_{k, 0}, i S_{\Upsilon, 0}\right] \widetilde{\varphi}\right\rangle=\left\langle\widetilde{\varphi},\left[\dot{\Delta}_{k, 0}, i S_{\Upsilon, 0}\right] \widetilde{\varphi}\right\rangle$.

We concentrate on the low energy part. We add (6.10) and (6.11) and infer

$\left\langle\widetilde{\varphi},\left[\dot{\Delta}_{k, 0}, i S_{\Upsilon, 0}\right] \widetilde{\varphi}\right\rangle=\left\langle\widetilde{\varphi},\left[\Delta_{k, 0}, i S_{\Upsilon, 0}\right] \widetilde{\varphi}\right\rangle$

$$
=\sum_{i=0,1}\left\langle\widetilde{\varphi}, 4\left(-\partial_{r}^{2}+V_{p_{i}}-V_{p_{i}}(\infty)+T_{\Upsilon}\right) \otimes P_{\operatorname{ker}\left(\Delta_{k-i}^{M}\right)} \widetilde{\varphi}\right\rangle+\left\langle\varphi, K_{2} \varphi\right\rangle
$$

for a certain $K_{2}=K_{2}(\Upsilon) \in \mathcal{K}\left(\mathcal{D}\left(\Delta_{k}\right), \mathcal{D}\left(\Delta_{k}\right)^{*}\right)$ and with $T_{\Upsilon}:=\partial_{r}\left(\partial_{r}+i \Phi_{\Upsilon}\right)$. The compactness of $K_{2}$ follows by noticing that $L_{0}^{-1} \in \mathcal{K}\left(\mathcal{D}\left(\Delta_{k, 0}\right), \mathscr{H}_{0}\right)$ by RellichKondrakov. We now control the size of $T_{\Upsilon}$. We have

$$
\left\|\widetilde{\mu}_{0} T_{\Upsilon}\left(1 \otimes P_{0}\right) \widetilde{\mu}_{0}\right\|_{\mathcal{B}\left(\mathcal{D}\left(\Delta_{k, 0}\right), \mathcal{D}\left(\Delta_{k, 0}\right)^{*}\right)} \text { tends to } 0 \text { as } \Upsilon \text { goes to infinity. }
$$

Indeed, $\widetilde{\mu}$ stabilizes $\mathcal{D}\left(\Delta_{k, 0}\right)$. Then $-\partial_{r}^{2} \widetilde{\mu}$ belongs to $\mathcal{B}\left(\mathcal{D}\left(\Delta_{k, 0}\right), L^{2}(\mathbb{R})\right)$ and $\left(-\partial_{r}^{2}+\right.$ $i)^{-2} T_{\Upsilon}$ tends to 0 in norm by functional calculus, as $\Upsilon$ goes to infinity.

We now extract some positivity. Let $\theta \in \mathcal{C}_{c}^{\infty}(\mathbb{R}, \mathbb{R})$ such that $\left.\theta\right|_{\mathcal{J}}=1$, such that $\kappa(p)$ is disjoint from the support of $\theta$ and such that the distance from $\mathcal{J}$ to the complement of the support is smaller than $c_{\mathcal{J}}$. If the support is lower than $\kappa(p)$, the Mourre estimate is trivial because $\theta\left(\Delta_{k}\right)$ is compact, by Proposition 5.2. We assume then that $\operatorname{supp}(\theta)$ is above inf $(\kappa(p))$. Using (6.13), the fact that $\mathcal{U} \theta\left(\dot{\Delta}_{k}\right) \mathcal{U}^{-1}$ stabilizes the decomposition (5.5), that $\left[\Delta_{k, 0}, i S_{\Upsilon, 0}\right]$ is bounded, we get 
that there is a compact operator $K_{0}$ in $\mathscr{H}_{0}$ so that:

$$
\begin{aligned}
\tilde{\mu}_{0} \mathcal{U}^{-1} \theta\left(\Delta_{k}\right) \mathcal{U}\left[\Delta_{k, 0}, i S_{\Upsilon, 0}\right] \mathcal{U} \theta\left(\Delta_{k, 0}\right) \mathcal{U}^{-1} \tilde{\mu}_{0} \\
=\tilde{\mu}_{0} P_{0} \mathcal{U}^{-1} \theta\left(\dot{\Delta}_{k}\right) \mathcal{U}\left[\dot{\Delta}_{k, 0}, i S_{\Upsilon, 0}\right] \mathcal{U} \theta\left(\dot{\Delta}_{k}\right) \mathcal{U}^{-1} P_{0} \tilde{\mu}_{0}+K_{0} .
\end{aligned}
$$

By ellipticity, we have

$$
\mathcal{U} \theta\left(\dot{\Delta}_{k}\right) \mathcal{U}^{-1} \tilde{\mu} P_{0} \mathscr{H}_{0} \subset \mathcal{C}_{0}^{\infty}((c, \infty)) \otimes P_{0} L^{2}\left(M, \Lambda^{k} M \oplus \Lambda^{k-1} M\right) ;
$$

i.e., the radial part tends to 0 at $c$ and at infinity. The analysis is reduced to the one of the Friedrichs extension $H_{i}^{F}$ of $H_{i}=-\partial_{r}^{2}+V_{p_{i}}$ on $L^{2}(c, \infty)$. Indeed, by stability of the decomposition (5.5), we have $\mathcal{U} \theta\left(\dot{\Delta}_{k}\right) \mathcal{U}^{-1}\left(1 \otimes P_{0}\right) \tilde{\mu}_{0}=\sum_{i=1,2} \theta\left(H_{i}^{F}\right) \otimes$ $P_{\text {ker }\left(\Delta_{k-i}^{M}\right)} \tilde{\mu}_{0}$.

Up to $T_{\Upsilon}$ and compact terms, the commutator of $H_{i}^{F}$ with $S_{\Upsilon, 0}$, given by (6.14), is $4 H_{i}^{F}-V_{p_{i}}(\infty)$. We have two possibilities: if the support of $\theta\left(H_{i}^{F}\right)$ is under $V_{p_{i}}(\infty)$, then for all $c \in \mathbb{R}$, there is a compact operator $K$ such that $\theta\left(H_{i}^{F}\right) H_{i}^{F} \theta\left(H_{i}^{F}\right)=c \theta\left(H_{i}^{F}\right)+K$, since the spectral measure is compact. If the support is above, then there is a compact $K$ such that $\theta\left(H_{i}^{F}\right)\left(H_{i}^{F}-V_{p_{i}}(\infty)\right) \theta\left(H_{i}^{F}\right) \geq$ $\inf \left(\operatorname{supp}(\theta)-V_{p_{i}}(\infty)\right) \theta\left(H_{i}^{F}\right)+K$. Adding back $T_{\Upsilon}$ and the compact part, we obtain

$$
\begin{aligned}
\tilde{\mu}_{0} P_{0} \mathcal{U} \theta\left(\dot{\Delta}_{k}\right) \mathcal{U}^{-1}\left[\Delta_{k, 0}, i S_{\Upsilon, 0}\right] \mathcal{U} \theta\left(\dot{\Delta}_{k}\right) \mathcal{U}^{-1} P_{0} \tilde{\mu}_{0} \geq 4 c_{\mathcal{J}} \tilde{\mu}_{0} P_{0} \mathcal{U} \theta^{2}\left(\dot{\Delta}_{k}\right) \mathcal{U}^{-1} P_{0} \tilde{\mu}_{0} \\
+\tilde{\mu}_{0} P_{0} \mathcal{U} \theta\left(\dot{\Delta}_{k}\right) \mathcal{U}^{-1} T_{\Upsilon} \mathcal{U} \theta\left(\dot{\Delta}_{k}\right) \mathcal{U}^{-1} P_{0} \tilde{\mu}_{0}+K_{0}
\end{aligned}
$$

We now remove the $P_{0}$. We add $P_{0}^{\perp} \theta\left(\dot{\Delta}_{k}\right) \tilde{\mu}_{0}$ on the right and on the left. This is a compact operator as it is equal to

$$
\left(\Delta_{k, 0}^{F} P_{0}^{\perp}+i\right)^{-1}\left(\Delta_{k, 0}^{F} P_{0}^{\perp}+i\right) \tilde{\mu}_{0} P_{0}^{\perp} \mathcal{U} \theta\left(\dot{\Delta}_{k}\right) \mathcal{U}^{-1} \tilde{\mu}_{0}+P_{0}^{\perp} \mu_{0} \mathcal{U} \theta\left(\dot{\Delta}_{k}\right) \mathcal{U}^{-1} \tilde{\mu}_{0},
$$

where $\Delta_{k, 0}^{F} P_{0}^{\perp}$ denotes the Friedrichs extension of $\Delta_{k, 0} P_{0}^{\perp}$. Its resolvent is compact by Proposition 5.1. Hence, the first term is compact as $\mu_{0} P_{0}^{\perp} \mathcal{U} \theta\left(\dot{\Delta}_{k}\right) \mathcal{U}^{-1} \tilde{\mu}_{0}$ is with value in $\mathcal{D}\left(\Delta_{k, 0}^{F} P_{0}^{\perp}\right)$. The compactness of the second terms follows from RellichKondrakov.

We get rid of the term in $T_{\Upsilon}$ and let $\varepsilon_{\Upsilon}$ appear by using the bound (6.15). Going back to the spectral measure of $\Delta_{k}$ with the help of (6.13), we infer that

$$
\tilde{\mu}_{0} \mathcal{U} \theta\left(\Delta_{k}\right) \mathcal{U}^{-1}\left[\Delta_{k, 0}, i S_{\Upsilon, 0}\right] \mathcal{U} \theta\left(\Delta_{k}\right) \mathcal{U}^{-1} \tilde{\mu}_{0} \geq 4\left(c_{\mathcal{J}}-\varepsilon_{\Upsilon}\right) \tilde{\mu}_{0} \mathcal{U} \theta^{2}\left(\Delta_{k}\right) \mathcal{U}^{-1} \tilde{\mu}_{0}+K_{0}
$$

holds true in $\mathscr{H}_{0}$ in the form sense. By Rellich-Kondrakov, up to more compactness, this implies (6.9) with $\theta$ instead of $E_{\mathcal{J}}$. To conclude, apply $E_{\mathcal{J}}\left(\Delta_{k}\right)$ on both sides.

6.4. The spectral and scattering theory. Directly applying the Mourre theory with Theorem 6.2 , one obtains the results $(1)-(4)$ of Theorem 1.3 with the metric $g$. The aim of the section is to complete the result and to go into perturbation theory so as to get the other metrics. In the earlier version of the Mourre theory for one self-adjoint operator $H$ having a spectral gap, one used something stronger than the hypothesis $H \in \mathcal{C}^{2}(A)$, where $A$ is the conjugate operator. This means that $\left[\left[(H+i)^{-1}, A\right], A\right]$ extends to a bounded operator. Keeping this hypothesis leads to a too-weak perturbation theory. In this section, we check a weak version of the two-commutators hypothesis. We say that $H \in \mathcal{C}^{1,1}(A)$ if

$$
\int_{0}^{1}\left\|\left[\left[(H+i)^{-1}, e^{i t A}\right], e^{i t A}\right]\right\| \frac{d t}{t^{2}}<\infty .
$$


If we have the invariance of the domain, $e^{i t A} \mathcal{D}(H) \subset \mathcal{D}(H)$, [1, Theorem 6.3.4] allows us to reformulate this condition in the equivalent

$$
\int_{0}^{1}\left\|\left[\left[H, e^{i t A}\right], e^{i t A}\right]\right\|_{\mathcal{B}\left(\mathcal{D}(H), \mathcal{D}(H)^{*}\right)} \frac{d t}{t^{2}}<\infty .
$$

This one is naturally named $\mathcal{C}^{1,1}\left(A ; \mathcal{D}(H), \mathcal{D}(H)^{*}\right)$. We refer to 1 for more properties. This is the optimal class of operators which give a limit absorption principle for $H$ in some optimal Besov spaces associated to $S_{\Upsilon}$. For the sake of simplicity, we will not formulate the result with these Besov spaces and keep the power of $\left\langle S_{\Upsilon}\right\rangle^{s}$. Those operators are replaced in the final result by $\langle L\rangle^{s}$ freely, using Lemma 6.1

The proof of Theorem 1.3 is given at the end of the section. The operator $\Delta_{k}$ belongs to $\mathcal{C}^{2}\left(S_{\Upsilon}\right)$ and therefore to $\mathcal{C}^{1,1}\left(S_{\Upsilon}\right)$. We have the invariance of the domain, by Lemma 6.4. Now take a symmetric relatively compact perturbation $T$ of $\Delta_{k}$ which lies in $\mathcal{C}^{1,1}\left(S_{\Upsilon} ; \mathcal{D}\left(\Delta_{k}\right), \mathcal{D}\left(\Delta_{k}\right)^{*}\right)$. Then a Mourre estimate holds for $\Delta+T$, and one gets (11)-(3) of Theorem 1.3 by using Theorem [1, Theorem 7.5.2]. The Hölder regularity of the resolvent, point (41), follows from [13); see the references therein. If the perturbation is short-range (see below) or local, then one has point (5) by using [1, Theorem 7.6.11]. by

We keep the notation of Section 6.1 Let $\partial_{L}$ be the closure of the operator defined

$$
\left(\partial_{L} f\right)(x, m)=x^{2-p}\left(\partial_{x} f\right)(x)-\alpha_{k} x^{1-p} f(x), \quad \text { with } \alpha_{k}=(n-2 k-1) p / 2,
$$

for $f \in \mathcal{C}_{c}^{\infty}\left(X^{\prime}, \Lambda^{k} X\right)$. Note that $\partial_{r}=\mathcal{U} \partial_{L} \mathcal{U}^{-1}$. Consider a symmetric differential operator $T: \mathcal{D}\left(\Delta_{k}\right) \rightarrow \mathcal{D}\left(\Delta_{k}\right)^{*}$. Let $\theta_{\text {sr }}$ be in $\mathcal{C}_{c}^{\infty}((0, \infty))$, not identically $0 ; T$ is short-range if

$$
\int_{1}^{\infty}\left\|\theta_{\mathrm{sr}}\left(\frac{L}{r}\right) T\right\|_{\mathcal{B}\left(\mathcal{D}\left(\Delta_{k}\right), \mathcal{D}\left(\Delta_{k}\right)^{*}\right)} d r<\infty
$$

and long-range if

$$
\begin{array}{r}
\int_{1}^{\infty}\left\|[T, L] \theta_{\operatorname{lr}}\left(\frac{L}{r}\right)\right\|_{\mathcal{B}\left(\mathcal{D}\left(\Delta_{k}\right), \mathcal{D}\left(\Delta_{k}\right)^{*}\right)}+\left\|\tilde{\mu}\left[T, P_{0}\right] L \theta_{\operatorname{lr}}\left(\frac{L}{r}\right) \tilde{\mu}\right\|_{\mathcal{B}\left(\mathcal{D}\left(\Delta_{k}\right), \mathcal{D}\left(\Delta_{k}\right)^{*}\right)} \\
+\left\|P_{0} \tilde{\mu}\left[T, \partial_{L}\right] L \theta_{\operatorname{lr}}\left(\frac{L}{r}\right) \tilde{\mu}\right\|_{\mathcal{B}\left(\mathcal{D}\left(\Delta_{k}\right), \mathcal{D}\left(\Delta_{k}\right)^{*}\right)} \frac{d r}{r}<\infty
\end{array}
$$

where $\theta_{\mathrm{lr}}$ is the characteristic function of $[1, \infty)$ in $\mathbb{R}$.

The first condition is evidently satisfied if there is an $\varepsilon>0$ such that

$$
\left\|L^{1+\varepsilon} T\right\|_{\mathcal{B}\left(\mathcal{D}\left(\Delta_{k}\right), \mathcal{D}\left(\Delta_{k}\right)^{*}\right)}<\infty
$$

and the second one if

$$
\begin{array}{r}
\left\|L^{\varepsilon}[T, L]\right\|_{\mathcal{B}\left(\mathcal{D}\left(\Delta_{k}\right), \mathcal{D}\left(\Delta_{k}\right)^{*}\right)}+\left\|L^{1+\varepsilon} \widetilde{\mu}\left[T, P_{0}\right] \widetilde{\mu}\right\|_{\mathcal{B}\left(\mathcal{D}\left(\Delta_{k}\right), \mathcal{D}\left(\Delta_{k}\right)^{*}\right)} \\
+\left\|L^{1+\varepsilon} \widetilde{\mu}\left[T, \partial_{L}\right] P_{0} \widetilde{\mu}\right\|_{\mathcal{B}\left(\mathcal{D}\left(\Delta_{k}\right), \mathcal{D}\left(\Delta_{k}\right)^{*}\right)}<\infty .
\end{array}
$$

To show that the first class is in $\mathcal{C}^{1,1}\left(S_{\Upsilon} ; \mathcal{D}\left(\Delta_{k}\right), \mathcal{D}\left(\Delta_{k}\right)^{*}\right)$, one uses [1, Theorem 7.5.8]. The hypotheses are satisfied thanks to Lemmata 6.1 and 6.10, This morally relies on the fact that the conjugate operator $S_{\Upsilon}$ is controlled by $L$. Note that (6.18) does not require the perturbation to be radial. Concerning the second class, it is enough to show that $\left[T, S_{\Upsilon}\right] \in \mathcal{C}^{0,1}\left(S_{\Upsilon} ; \mathcal{D}\left(\Delta_{k}\right), \mathcal{D}\left(\Delta_{k}\right)^{*}\right)$, i.e.

$$
\int_{0}^{1}\left\|\left[\left[T, S_{\Upsilon}\right], e^{i t S_{\Upsilon}}\right]\right\|_{\mathcal{B}\left(\mathcal{D}\left(\Delta_{k}\right), \mathcal{D}\left(\Delta_{k}\right)^{*}\right)} \frac{d t}{t}<\infty .
$$


This follows by using [1, Proposition 7.5.7] (see the proof of [1, Proposition 7.6.8], for instance). This also morally relies on the fact that the conjugate operator $S_{\Upsilon}$ is controlled by $L$. Unlike for the previous class, the commutator $\left[T, S_{\Upsilon}\right]$ appears and one has to take into account the component coming from $P_{0}$ for instance. The condition involving $\left[P_{0}, T\right]$ in (6.19) essentially tells us that the non-radial part of $T$ is a short-range perturbation. This is why we will ask the long-range perturbation to be radial.

Straightforwardly, we get the short- and long-range perturbations of the potential.

Lemma 6.7. Let $V \in L^{\infty}(X)$. If $\left\|L^{1+\varepsilon} V\right\|_{\infty}<\infty$, then the perturbation $V$ is short-range in $\mathcal{B}(\mathscr{H})$. If $V$ is radial, tends to 0 as $x \rightarrow 0$ and $\left\|L^{1+\varepsilon} x^{2-p} \partial_{x} V\right\|_{\infty}<$ $\infty$, then $V$ is a long-range perturbation in $\mathcal{B}(\mathscr{H})$.

In this section we will prove the points (2) - (5) of Theorem 1.3 for the conformally perturbed metric $\tilde{g}:=\left(1+\rho_{\mathrm{sr}}+\rho_{\mathrm{lr}}\right) g_{p}^{\text {model }}$, where $\rho_{\mathrm{sr}}$ and $\rho_{\text {lr }}$ are in $\mathcal{C}^{\infty}(X)$, $\inf _{x \in X}\left(1+\rho_{\mathrm{sr}}(x)+\rho_{\mathrm{lr}}(x)\right)>1, \rho_{\mathrm{lr}}$ is radial, where $g_{p}^{\text {model }}$ is given by (5.3) and where we assume

$$
\begin{array}{r}
\left\|L^{1+\varepsilon} \rho_{\mathrm{sr}}\right\|_{\infty}+\left\|d \rho_{\mathrm{sr}}\right\|_{\infty}+\left\|\Delta \rho_{\mathrm{sr}}\right\|_{\infty}<\infty, \\
\left\|L^{\varepsilon} \rho_{\mathrm{lr}}\right\|_{\infty}+\left\|L^{1+\varepsilon} x^{2-p} \partial_{x} \rho_{\mathrm{lr}}\right\|_{\infty}+\left\|\Delta \rho_{\mathrm{lr}}\right\|_{\infty}<\infty .
\end{array}
$$

Lemma 6.11 shows that the Laplacian associated to the metric $\rho_{\mathrm{sr}}\left(\rho_{\mathrm{lr}}\right.$ resp.) is a short-range perturbation (long-range resp.) of the one given by the metric $g_{p}^{\text {model }}$ in some precise sense.

Remark 6.8. For the sake of simplicity, we treat only conformal perturbations of the metric. However, there is no obstruction in our method to treat more general perturbations as in [10. The only fact we really use is that the domain of the perturbed Laplacian is the same as that of the free Laplacian. In the general case, we need to impose an extra condition (A.2). In the case of a conformal change, this follows automatically by the condition (6.20) on the derivatives.

We define the Sobolev spaces associated to the Laplacian given by the metric g. Set $\mathscr{H}^{s}:=\mathcal{D}\left((1+\Delta)^{s / 2}\right)=\mathcal{D}\left(|d+\delta|^{s}\right)$ for $s \geq 0$, and $\mathscr{H}^{s}:=\left(\mathscr{H}^{-s}\right)^{*}$ for $s$ negative (here we use the Riesz isomorphism between $\mathscr{H}:=\mathscr{H}^{0}$ and $\mathscr{H}^{*}$ ). We will use $\mathscr{H}^{s}$ with $s \in[-2,2]$; these spaces are equal to the ones obtained using the metric $\tilde{g}$ since the norms are equivalent.

To analyze the perturbation of the metric, unlike in [16, we work simultaneously with forms of all degrees. Since $L$ stabilizes the space of $k$-forms, one gets $\mathcal{C}^{1,1}$ regularity for $\Delta_{k}$ by showing it for $\Delta$.

We now proceed in two steps and set $g_{\mathrm{lr}}:=\left(1+\rho_{\mathrm{lr}}\right) g_{p}^{\text {model }}$. We define $U_{\mathrm{lr}}$ and $U_{\mathrm{sr}}$ to be the operators of multiplication by $\left(1+\rho_{\mathrm{lr}}\right)^{(n-2 k) / 4}$ and $\left(1+\rho_{\mathrm{sr}} /(1+\right.$ $\left.\left.\rho_{\mathrm{lr}}\right)\right)^{(n-2 k) / 4}$, respectively, when restricted to $k$-forms. Observe that $\rho_{\mathrm{sr}} /\left(1+\rho_{\mathrm{lr}}\right)$ is short range in the sense of (6.20). Now note that the transformations

$$
\begin{aligned}
& U_{\mathrm{lr}}: L^{2}\left(X, \Lambda^{*} X, g\right) \longrightarrow L^{2}\left(X, \Lambda^{*} X, g_{\mathrm{lr}}\right):=\mathscr{H}_{\mathrm{lr}}, \\
& U_{\mathrm{sr}}: L^{2}\left(X, \Lambda^{*} X, g_{\mathrm{lr}}\right) \longrightarrow L^{2}\left(X, \Lambda^{*} X, \tilde{g}\right):=\mathscr{H}_{\tilde{g}}
\end{aligned}
$$

are unitary. Also set $U_{\tilde{g}}:=U_{\mathrm{sr}} U_{\mathrm{lr}}$.

Lemma 6.9. The operators $L^{1+\varepsilon}\left(U_{\mathrm{sr}}-1\right), L^{\varepsilon}\left(U_{\mathrm{lr}}-1\right)$ and $L^{1+\varepsilon}\left[U_{\mathrm{lr}}, \partial_{L}\right]$ extend to bounded operators in $\mathscr{H}$. 
Proof. We concentrate on the short-range case. Easily, it is enough to show that, for all non-negative integers $\alpha, L^{1+\varepsilon}\left(\left(1+\rho_{\mathrm{sr}} /\left(1+\rho_{\mathrm{lr}}\right)\right)^{\alpha}-1\right)$ is bounded. One shows this by using (6.20) and the binomial formula. For the first part of the long-range case, we do the same. The second part is obvious using the chain-rule and again (6.20).

We gather various technicalities concerning the operator $L$. By taking the adjoint and as the spaces $\mathscr{H}^{s}$ are independent of $\rho_{\mathrm{sr}}$ and $\rho_{\mathrm{lr}}$, one gets similar bounds for $\delta_{g}, \delta_{g_{\mathrm{lr}}}$ and $\delta_{\tilde{g}}$. Note that we add the metric in the subscript to stress the dependence on the metric.

Lemma 6.10. Let $s \in[-2,2]$ and $\alpha \geq 0$.

(1) The operator of multiplication by $d L$ is bounded in $\mathscr{H}$. The support of $d L$ is in $(0, \varepsilon) \times M$ and $d L=-x^{p-2} d x$ for $x$ small enough.

(2) $e^{i t L} \mathscr{H}^{2} \subset \mathscr{H}^{2}$ and $\left\|e^{i t L}\right\|_{\mathcal{B}\left(\mathscr{H}^{2}\right)} \leq c\left(1+t^{2}\right)$.

(3) $L^{-\alpha}\left[d, L^{1+\alpha}\right]$ is bounded in $\mathscr{H}$ and $L^{-\alpha}\left[\Delta_{\tilde{g}}, L^{1+\alpha}\right]$ is bounded in $\mathcal{B}\left(\mathscr{H}^{1}, \mathscr{H}\right)$.

(4) $\tilde{\mu}\left[L^{1+\alpha},\left[d, \partial_{L}\right]\right] L^{-\alpha} P_{0} \tilde{\mu}$ and $\tilde{\mu} L^{-\alpha}\left[L^{1+\alpha},\left[d, \partial_{L}\right]\right] P_{0} \tilde{\mu}$ are bounded in $\mathscr{H}^{s}$.

(5) $\tilde{\mu} L\left[d, \partial_{L}\right] P_{0} \tilde{\mu}$ and $\tilde{\mu}\left[d, \partial_{L}\right] L P_{0} \tilde{\mu}$ are bounded from $\mathscr{H}^{s}$ to $\mathscr{H}^{s-1}$.

Proof. The first point is obvious. The next two points follow by noticing that single and double commutators of $L$ with $d$ or $\delta_{g}$ are bounded in $\mathscr{H}$. Indeed, we recall that $L_{0}$ is the multiplication by $r$ above $X_{0}^{\prime}$. One derives the result for $\delta_{\tilde{g}}$ by conjugating by $U_{\tilde{g}}$. For the last two points, it is enough to take $\alpha=2$ by interpolation and duality. A straightforward computation gives that for a smooth $k$-form $f$ with compact support on the cusp, we have:

$$
\left[d, \partial_{L}\right] P_{0} f=\left(2(1-p) x^{1-p} d+\alpha_{k}(1-p) x^{2(1-p)} x^{p-2} d x \wedge\right) P_{0} f_{1},
$$

where $f=f_{1}+x^{-2} d x \wedge f_{2}$, following (3.2). Here, we used $d P_{0}=\left(d x \wedge \partial_{x} \cdot\right) P_{0}$. Hence the fourth point is clear. For the last one, note that $L x^{(1-p)}$ is constant on the cusp, for $p<1$.

Lemma 6.11. We set $W_{\mathrm{lr}}:=U_{\mathrm{lr}}^{-1} \Delta_{\mathrm{lr}} U_{\mathrm{lr}}$ and $D_{U_{\mathrm{lr}}}:=d+U_{\mathrm{lr}} \delta U_{\mathrm{lr}}^{-1}$ in $\mathscr{H}_{g}$ and we also set $W_{\mathrm{sr}}:=U_{\mathrm{sr}}^{-1} \Delta_{\mathrm{sr}} U_{\mathrm{sr}}$ and $D_{U_{\mathrm{sr}}}:=d+U_{\mathrm{sr}} \delta U_{\mathrm{sr}}^{-1}$ in $\mathscr{H}_{g_{\mathrm{lr}}}$. Then:

(1) We have $W_{\mathrm{lr}}=U_{\mathrm{lr}} D_{U_{\mathrm{lr}}}^{2} U_{\mathrm{lr}}^{-1}$ and $W_{\mathrm{sr}}=U_{\mathrm{sr}} D_{U_{\mathrm{sr}}}^{2} U_{\mathrm{sr}}^{-1}$. Moreover, they are essentially self-adjoint on $\mathcal{C}_{c}^{\infty}\left(X, \Lambda^{*} X\right)$ in $\mathscr{H}_{g}$ and $\mathscr{H}_{\mathrm{l}}$, respectively. Their domain is $\mathscr{H}^{2}$.

(2) We have $\left(W_{\mathrm{lr}}+i\right)^{-1}-\left(\Delta_{g}+i\right)^{-1} \in \mathcal{K}\left(\mathscr{H}_{g}\right)$ and $\left(W_{\mathrm{sr}}+i\right)^{-1}-\left(\Delta_{\mathrm{lr}}+i\right)^{-1} \in$ $\mathcal{K}\left(\mathscr{H}_{\mathrm{lr}}\right)$.

(3) The operator $W_{\mathrm{lr}}$ is a long-range perturbation of $\Delta_{g}$ inside $\mathcal{B}\left(\mathscr{H}^{2}, \mathscr{H}^{-2}\right)$.

(4) The operator $W_{\mathrm{sr}}$ is a short-range perturbation of $\Delta_{\mathrm{lr}}$ inside $\mathcal{B}\left(\mathscr{H}^{2}, \mathscr{H}^{-2}\right)$.

Proof. Since the manifold is complete, $D_{U_{\mathrm{lr}}}$ is self-adjoint on the closure of $\mathcal{C}_{c}^{\infty}\left(X, \Lambda^{*} X\right)$ under the norm $\|f\|+\|d f\|+\left\|\delta_{g_{\mathrm{lr}}} f\right\|$. Now remark that for all $\alpha \in \mathbb{R}$, $\left(1+\rho_{\mathrm{lr}}\right)^{\alpha}$ stabilizes $\mathscr{H}^{2}$ and, by duality and interpolation, $\mathscr{H}^{s}$ for all $s \in[-2,2]$. Note also that $\mathcal{D}\left(U_{\mathrm{lr}} D_{\mathrm{lr}} U_{\mathrm{lr}}^{-1}\right)=\mathcal{D}(D)$ to get the first point (the argument being the same for the short-range part).

We now compare the two operators $F_{l r}:=W_{\mathrm{lr}}-\Delta_{g}$ and $F_{\mathrm{sr}}:=W_{\mathrm{sr}}-\Delta_{g_{\mathrm{lr}}}$ in $\mathscr{H}_{g}$ and in $\mathscr{H}_{g_{\mathrm{lr}}}$, respectively. We compute on $\mathcal{C}_{c}^{\infty}\left(X, \Lambda^{*} X\right)$,

$$
F .=U \cdot\left(D_{U}-D\right) D_{U} U_{.}^{-1}+U \cdot D\left(D_{U} \cdot-D\right) U^{-1}+U \cdot D^{2}\left(U^{-1}-1\right)+(U .-1) D^{2} .
$$


Also note that $D_{U} .-D=(U .-1) d^{*} U_{.^{-1}}-d^{*}\left(U^{-1}-1\right)$, where the adjoint of $d$ is taken in $\mathscr{H}_{g}$ and in $\mathscr{H}_{g_{\mathrm{lr}}}$, respectively.

To get point (2), since $W_{\mathrm{lr}}$ and $\Delta_{g}$ have the same domain, it is enough to show that $W_{\text {lr }}-\Delta_{g}$ is a compact operator in $\mathcal{B}\left(\mathscr{H}^{2}, \mathscr{H}^{-2}\right)$ by writing the difference of the resolvent in a generalized way; see [16, Lemma 6.13]. This follows using $U^{ \pm 1}-1 \in \mathcal{K}\left(\mathscr{H}^{1}, \mathscr{H}\right)$. The argument is the same as for the short-range part.

Focus on point (4). We treat one of the bad terms. We compute on $\mathcal{C}_{c}^{\infty}\left(X, \Lambda^{*} X\right)$ :

$$
\begin{aligned}
L^{1+\varepsilon} \delta_{\mathrm{lr}}\left(U_{\mathrm{sr}}^{-1}-1\right) D_{U_{\mathrm{sr}}} U_{\mathrm{sr}}^{-1}= & \delta_{\mathrm{lr}} L^{1+\varepsilon}\left(U_{\mathrm{sr}}^{-1}-1\right) D_{U_{\mathrm{sr}}} U_{\mathrm{sr}}^{-1} \\
& +\left(\left[L^{1+\varepsilon}, \delta_{\mathrm{lr}}\right] L^{-\varepsilon}\right) L^{\varepsilon}\left(U_{\mathrm{sr}}^{-1}-1\right) D_{U_{\mathrm{sr}}} U_{\mathrm{sr}}^{-1} .
\end{aligned}
$$

By density, the first term extends to an element of $\mathcal{B}\left(\mathscr{H}^{1}, \mathscr{H}^{-1}\right)$. Indeed, using the invariance of the domains, $D_{U_{\mathrm{sr}}} U_{\mathrm{sr}}^{-1} \in \mathcal{B}\left(\mathscr{H}^{1}, \mathscr{H}\right)$ and $L^{1+\varepsilon}\left(U_{\mathrm{sr}}^{-1}-1\right) \in \mathcal{B}(\mathscr{H})$ by Lemma 6.9. The second term extends to an element of $\mathcal{B}\left(\mathscr{H}^{1}, \mathscr{H}\right)$ using Lemma 6.10. The other terms are treated in the same way.

We turn to point (3). We treat as above the term in $L^{\varepsilon}$. It remains to show that $\left\|L^{1+\varepsilon} \tilde{\mu}\left[F_{\mathrm{lr}}, \partial_{L}\right] P_{0} \tilde{\mu}\right\|_{\mathcal{B}\left(\mathscr{H}^{2}, \mathscr{H}^{-2}\right)}$ is finite. We pick a bad term and drop $P_{0}, \tilde{\mu}$ for clarity:

$$
\begin{aligned}
& L^{1+\varepsilon}\left[\delta\left(U_{\mathrm{lr}}^{-1}-1\right) D_{U_{\mathrm{lr}}} U_{\mathrm{lr}}^{-1}, \partial_{L}\right]=L^{1+\varepsilon}\left[\delta, \partial_{L}\right]\left(U_{\mathrm{lr}}^{-1}-1\right) D_{U_{\mathrm{lr}}} U_{\mathrm{lr}}^{-1} \\
& \quad+L^{1+\varepsilon} \delta\left[U_{\mathrm{lr}}^{-1}, \partial_{L}\right] D_{U_{\mathrm{lr}}} U_{\mathrm{lr}}^{-1}+L^{1+\varepsilon} \delta\left(U_{\mathrm{lr}}^{-1}-1\right)\left[D_{U_{\mathrm{lr}}}, \partial_{L}\right] U_{\mathrm{lr}}^{-1} \\
& \quad+L^{1+\varepsilon} \delta\left(U_{\mathrm{lr}}^{-1}-1\right) D_{U_{\mathrm{lr}}}\left[U_{\mathrm{lr}}^{-1}, \partial_{L}\right] .
\end{aligned}
$$

After commutation, the first term on the right-hand side becomes

$$
\left(\left[\delta, \partial_{L}\right] L\right)\left(L^{\varepsilon}\left(U_{\mathrm{lr}}^{-1}-1\right)\right) D_{U_{\mathrm{lr}}} U_{\mathrm{lr}}^{-1}+\left(\left[L^{1+\varepsilon},\left[\delta, \partial_{L}\right]\right] L^{-\varepsilon}\right)\left(L^{\varepsilon}\left(U_{\mathrm{lr}}^{-1}-1\right)\right) D_{U_{\mathrm{lr}}} U_{\mathrm{lr}}^{-1}
$$

while the second one is

$$
\delta\left(L^{1+\varepsilon}\left[U_{\mathrm{lr}}^{-1}, \partial_{L}\right]\right) D_{U_{\mathrm{lr}}} U_{\mathrm{lr}}^{-1}+\left(\left[L^{1+\varepsilon}, \delta\right] L^{-\varepsilon}\right)\left(L^{\varepsilon}\left[U_{\mathrm{lr}}^{-1}, \partial_{L}\right]\right) D_{U_{\mathrm{lr}}} U_{\mathrm{lr}}^{-1} .
$$

Use Lemma 6.10 and Lemma 6.9 to control the terms in brackets. They are elements of $\mathcal{B}\left(\mathscr{H}^{1}, \mathscr{H}^{-1}\right)$ by density. The other terms are controlled in the same way; by commutation, we let $L^{\varepsilon}$ touch $\left(U_{\mathrm{lr}}^{ \pm 1}-1\right)$ and $L^{1+\varepsilon}$ touch $\left[U_{\mathrm{lr}}^{ \pm 1}, \partial_{L}\right]$.

We are now in a position to show the Mourre estimate for the perturbed metric.

Proof of Theorem 1.3. Let $\mathcal{J}$ be an interval containing no element of $\kappa(p)$. Taking $\Upsilon$ large enough, Theorem 6.2 gives $c>0$ and a compact operator $K$ so that the Mourre estimate

$$
E_{\mathcal{J}}(T)[T, i S] E_{\mathcal{J}}(T) \geq c E_{\mathcal{J}}(T)+K
$$

holds in the sense of forms in $L^{2}\left(X, \Lambda^{k} X, g\right)$, for $T=\Delta_{k}$. Moreover, $\Delta_{k}$ belongs to $\mathcal{C}^{2}\left(S ; \mathcal{D}\left(\Delta_{k}\right), L^{2}\left(X, \Lambda^{k} X, g\right)\right)$. We dropped the subscript $\Upsilon$ of $S$ as it plays no further rôle.

By Lemma 6.11. $W_{\text {lr }}$ belongs to the class $\mathcal{C}^{1,1}\left(S ; \mathcal{D}\left(\Delta_{k}\right), \mathcal{D}\left(\Delta_{k}\right)^{*}\right)$. In particular, we obtain $W_{\mathrm{lr}} \in \mathcal{C}_{\mathrm{u}}^{1}\left(S ; \mathcal{D}\left(\Delta_{k}\right), \mathcal{D}\left(\Delta_{k}\right)^{*}\right)$, meaning that $t \mapsto e^{i t S} W_{\mathrm{lr}} e^{-i t S}$ is norm continuous in $\mathcal{B}\left(\mathcal{D}\left(\Delta_{k}\right), \mathcal{D}\left(\Delta_{k}\right)^{*}\right)$. By the point (2) of Lemma 6.11] and [1, Theorem 7.2.9] the inequality (6.22) holds for $T=W_{\text {lr }}$ (up to changing $c$ and $K$ ). Now repeat the argument for the short-range part $W_{\mathrm{er}}$ and get the Mourre estimate with $T=U_{\mathrm{lr}}^{-1} W_{\mathrm{sr}} U_{\mathrm{lr}}$.

Now go into $\mathscr{H}_{\tilde{g}}$ conjugating with $U_{\tilde{g}}$. The conjugate operator becomes $S_{\tilde{g}}$. Therefore, $\Delta_{\tilde{g}, k} \in \mathcal{C}^{1,1}\left(S_{\tilde{g}} ; \mathcal{D}\left(\Delta_{\tilde{g}, k}\right), \mathcal{D}\left(\Delta_{\tilde{g}, k}\right)^{*}\right)$ and there are $c>0$ and a compact 
operator $K$ so that

$$
E_{\mathcal{J}}\left(T_{\tilde{g}}\right)\left[T_{\tilde{g}}, i S_{\tilde{g}}\right] E_{\mathcal{J}}\left(T_{\tilde{g}}\right) \geq c E_{\mathcal{J}}\left(T_{\tilde{g}}\right)+K
$$

holds in the sense of forms in $L^{2}\left(X, \Lambda^{k} X, \tilde{g}\right)$ for $T_{\tilde{g}}=\Delta_{\tilde{g}, k}$. We now add the perturbation given by $V_{\mathrm{sr}}$ and $V_{\mathrm{lr}}$. Note that $H_{0}:=\Delta_{\tilde{g}, k}+V_{\mathrm{lr}}$ has the same domain as $H:=H_{0}+V_{\mathrm{sr}}$ and that $(H+i)^{-1}-\left(H_{0}+i\right)^{-1}$ is compact by the RellichKondrakov lemma. By Lemma 6.7, we obtain $H \in \mathcal{C}^{1,1}\left(S_{\tilde{g}} ; \mathcal{D}(H), \mathcal{D}(H)^{*}\right)$. As above, the inequality (6.23) is true for $\tilde{T}=H$.

We now deduce the different claims of the theorem. The first comes from [1, Theorem 7.5.2]. The second one is a consequence of the Virial Lemma. For the third point first note that $\mathscr{L}_{s} \subset \mathcal{D}\left(|S|^{s}\right)$ for $s \in[0,2]$ by Lemma 6.1] and use [13. Finally, the last point follows from [1, Theorem 7.6.11].

We finish this section with two remarks which improve the result.

Remark 6.12. Concerning the point (2), we are able to show that the eigenvalues in $\kappa(p)$ are of finite multiplicity only in the case of the metric (2.1), i.e. when the perturbation is smooth, using [16, Lemma B.1]. At every other energy level, it follows from the Mourre estimate (6.23), with $\tilde{T}=H$, via the Virial Lemma.

Remark 6.13. If $M$ is disconnected and if one of its connected components $M_{0}$ has Betti numbers $b_{k}\left(M_{0}\right)=b_{k-1}\left(M_{0}\right)=0$, then by taking $L$ to be 1 on the corresponding cusp $[0, \infty) \times M_{0}$, any potential with support in this cusp and tending to 0 at infinity (without any required speed) is a short-range perturbation; see [16] for similar statements.

\section{BetTI NUMBERS AND CUSPS OF HYPERBOLIC MANIFOLDS}

We conclude by some relations between our analysis and the topology of finitevolume hyperbolic manifolds. Recall that the Betti numbers $b_{k}$ of the torus $T^{n}:=$ $\left(S^{1}\right)^{n}$ equal the binomial coefficients $\left(\begin{array}{l}n \\ k\end{array}\right)$; in particular, none of them (in the range $0, \ldots, n)$ is zero.

It is easy to see that a cylinder $M \times(0, \infty)$, with the metric $g_{1}$ given on the whole cylinder by (1.1) for $p=1$, is hyperbolic (i.e., it has constant sectional curvature -1 ) if and only if $M$ is flat (i.e., its sectional curvatures vanish identically). Moreover, every finite-volume complete hyperbolic manifold is of this form outside a compact set [4. Closed flat manifolds, also called Bieberbach manifolds, have been classified in dimension 3 by Hantzsche and Wendt 21. They obtain ten different topological types of Bieberbach manifolds, of which $\mathfrak{A}_{1}-\mathfrak{A}_{6}$ are orientable, while $\mathfrak{B}_{1}-\mathfrak{B}_{4}$ are non-orientable. We remark here that from the description of [21, page 610], it follows that $\mathfrak{A}_{6}$ is the only Bieberbach 3-manifold with first Betti number equal to 0. Since $\mathfrak{A}_{6}$ is orientable, by Poincaré duality we see that $b_{2}\left(\mathfrak{A}_{6}\right)=b_{1}\left(\mathfrak{A}_{6}\right)=0$; hence $\mathfrak{A}_{6}$ is a rational homology sphere. Its first integer homology group is isomorphic to $(\mathbb{Z} / 4 \mathbb{Z})^{3}$. Also from loc. cit. and from the explicit description of the holonomy groups in [40, the only non-orientable Bieberbach 3-manifold with second Betti number $b_{2}$ equal to 0 is $\mathfrak{B}_{4}$, which moreover has $b_{3}\left(\mathfrak{B}_{4}\right)=0$ by non-orientability.

As a by-product of our analysis we obtain the following Hodge decomposition on asymptotically hyperbolic manifolds.

Proposition 7.1. Let $(X, g)$ be a complete non-compact hyperbolic manifold of finite volume. Let $M$ be the boundary at infinity. If $n=\operatorname{dim}(X)$ is odd, also 
suppose that the Betti number $b_{\frac{n-1}{2}}(M)=0$. Then $\operatorname{Im}(d)$ and $\operatorname{Im}\left(\delta_{\tilde{g}}\right)$ are closed and

$$
L^{2}\left(X, \Lambda^{*} X\right)=\operatorname{ker}\left(\Delta_{\tilde{g}}\right) \oplus \operatorname{Im}(d) \oplus \operatorname{Im}\left(\delta_{\tilde{g}}\right) .
$$

The conclusion holds more generally for any metric $\tilde{g}$ which satisfies (A.1) and (A.2) with respect to the given hyperbolic metric.

This proposition is a consequence of the fact that 0 is not in the essential spectrum of the Hodge Laplacian (compare with 28]) and of a result of stability of the essential spectrum obtained in [14; see Proposition A.1.

Proof. Every complete non-compact hyperbolic manifold of finite volume is of the form (1.1) with $p=1$ outside a compact set. By Proposition 5.2, we obtain that $\Delta$ has 0 in its essential spectrum if and only if $n$ is odd and $b_{(n-1) / 2}$ is non-zero. Therefore, by hypothesis, 0 is not in the essential spectrum of the Hodge Laplacian $\Delta$. Now by Proposition A.1, 0 is not in the essential spectrum of $\Delta_{\tilde{g}}$. Using the closed graph theorem, it follows easily that $\operatorname{Im}(\Delta)$ is closed. Since the metric is smooth, one obtains the closedness of $\operatorname{Im}(d)$ and of $\operatorname{Im}\left(\delta_{\tilde{g}}\right)$ and also the announced Hodge decomposition; see [6. Theorem 5.10].

An important geometric question in dimension 4 is to decide which flat manifolds occur as cusps of hyperbolic manifolds. It is known that every manifold in the Hantzsche-Wendt list appears among the cusps of some finite-volume hyperbolic manifold [34, but it is not known whether 1-cusped hyperbolic 4-manifolds exist at all. There is an obstruction to the existence of oriented hyperbolic 4-manifolds with certain combinations of cusps. This obstruction, discovered by Long and Reid [25, is the integrality of the eta invariant (for the signature operator) of the oriented Bieberbach manifold modeling the cusps. The eta invariant of Dirac operators on Bieberbach 3-manifolds was computed by Pfäffle [36] and may provide additional obstructions. See also [18 and the references therein for an introduction to the eta invariant.

The essential spectrum of the Laplacian on $k$-forms was computed in 28, Theorem 1.11]; in particular, it is stated to be non-empty for all $k$. One implicit assumption in the proof of [28, Lemma 5.26] seems to be the non-vanishing of $H^{k}(M)$; the proof also works provided $H^{k-1}(M) \neq 0$. Rafe Mazzeo confirmed to us in a private communication that [24, Theorem 1.11] holds under the tacit assumption that the manifold $X$ is orientable, and that the cusp cross section $M$ has non-vanishing cohomology in degrees $k$ or $k-1$. However there exist flat 3-manifolds $M$ for which $b_{2}(M)=b_{1}(M)=0$, of the type $\mathfrak{A}_{6}$ in the Hantzsche-Wendt classification. By taking Cartesian products of such an $M$ with itself, we get examples in dimension $3 j$ for all $j \geq 1$. Thus we are led to the following.

Question 1. Does there exist a complete finite-volume hyperbolic manifold of dimension 4 such that each component of its boundary at infinity is the rational homology sphere $\mathfrak{A}_{6}$ ?

As we see in Theorem 1.2, this issue is crucial for the nature of the spectrum of the Laplacian on $k$-forms. In light of Theorem 1.2, such a manifold would provide a counterexample to [28, Theorem 1.11] for $k=2$. If we accepted the result of [28] to continue to hold as stated there in full generality, then the answer would always 
be negative; however, this is unlikely and we conjecture that there indeed exists such a 4-manifold. More generally, we are led to

Question 2. Does there exist a complete finite-volume hyperbolic manifold such that for some $k \in \mathbb{N}$, the boundary at infinity $M$ satisfies $b_{k}(M)=b_{k-1}(M)=0$ ?

This question we can answer affirmatively. In the literature there exists a nonorientable, finite-volume hyperbolic 4-manifold with 5 cusps, all of which are topologically of type $\mathfrak{B}_{4}$. It is obtained by gluing together the sides of a regular ideal 24-cell in hyperbolic 4-space in a particular way. There are 1171 such identifications possible [37]; one of them, called $\mathfrak{R T}_{1080}$ here ([37, Table 3, nr. crit. 1080]), has the desired property of having all its cusps of type $\mathfrak{B}_{4}$. It follows from Theorem 1.2 that the Laplacians on 3- and 4-forms on $\mathfrak{R T}_{1080}$ have purely discrete spectra, which shows that the additional hypothesis that at least one of $b_{k}(M), b_{k-1}(M)$ be different from 0 is necessary for [28, Theorem 1.1] to hold as stated. We remark here that Mazzeo and Phillips use the Hodge star operator, which only exists on oriented manifolds, to reduce the analysis to $k \leq n / 2$, while our counterexample is non-orientable and works for $n=4$ and $k \in\{3,4\}$.

In conclusion, the complete general statement about the essential spectrum of the Laplacian on forms on finite-volume hyperbolic manifolds, without any restriction on the cusp cross section, is obtained from [28, Theorem 1.11] by adding the following exceptional case, which follows from the above counterexample and our Theorem 1.2 ,

In the case where the volume is finite and all the cusps are modeled on Bieberbach manifolds with zero Betti numbers in dimensions $k$ and $k-1$, the spectrum of the Laplacian on $k$-forms is purely discrete and obeys the classical Weyl law.

The list from [37] includes precisely two hyperbolic 4-manifolds with all cusps of the same type in the Hantzsche-Wendt classification. One is $\mathfrak{R T}_{1080}$ mentioned above; the other one is $\mathfrak{R T}_{1011}$, whose five cusps are all of type $\mathfrak{B}_{1}$. It is a fact that $\mathfrak{B}_{1}$ has non-zero Betti numbers $b_{1}$ and $b_{2}$. Except for $\mathfrak{R} \mathfrak{T}_{1080}$, none of the Rattcliffe-Tschantz manifolds satisfies $b_{2}(M)=0$; therefore the corresponding Laplacians $\Delta_{2}$ and $\Delta_{3}$ have non-empty continuous spectrum (Theorem 1.3). The same is always true for $k=0,1$ since $b_{0}(M)$ is non-zero. However, $\Delta_{4}$ does have a purely discrete spectrum for quite a few manifolds from [37, Tables 3,4]. This is because on one hand, $b_{4}$ of a 3 -manifold is always 0 ; on the other hand, $b_{3}$ of a non-orientable 3-manifold is equally zero. Thus the hypothesis of Theorem 1.2 is satisfied for $k=4$ for every Rattcliffe-Tschantz manifold whose cusps are modeled on non-orientable Bieberbach manifolds. Besides $\mathfrak{R T} 1080$ these are: $\mathfrak{R T}_{35}, \mathfrak{R T}_{130}-\mathfrak{R T}_{156}, \mathfrak{R T}_{426}-\mathfrak{R T}_{516}, \mathfrak{R T}_{538}-\mathfrak{R T}_{544}, \mathfrak{R T}_{811}-\mathfrak{R T}_{865}, \mathfrak{R T}_{942}-\mathfrak{R T} \mathfrak{T}_{959}$, $\mathfrak{R T}_{1012}-\mathfrak{R T}_{1014}, \mathfrak{R T}_{1080}-\mathfrak{R T} \mathfrak{T}_{1084}, \mathfrak{R T}_{1095}, \mathfrak{R T}_{1101}-\mathfrak{R T}_{1105}, \mathfrak{R T}_{1109}, \mathfrak{R T}_{1122^{-}}$ $\mathfrak{R T} \mathfrak{T}_{1127}, \mathfrak{R T}_{1134}, \mathfrak{R T} \mathfrak{T}_{1142}, \mathfrak{R T} \mathfrak{T}_{1143}, \mathfrak{R T}_{1156}-\mathfrak{R T}_{1158}$, and $\mathfrak{R T} \mathfrak{T}_{1167}$. None of the above examples is orientable. Nevertheless, we expect the answer to Question 1 to be affirmative.

\section{Appendix A. Stability of the essential spectrum}

If $A, B$ are closed operators acting in a Hilbert space $\mathscr{H}$ and if there is $z \in$ $\rho(A) \cap \rho(B)$ such that $(A-z)^{-1}-(B-z)^{-1}$ is a compact operator, then we say that $B$ is a compact perturbation of $A$. If this is the case, then the difference 
$(A-z)^{-1}-(B-z)^{-1}$ is a compact operator for all $z \in \rho(A) \cap \rho(B)$. In particular, if $B$ is a compact perturbation of $A$, then $A$ and $B$ have the same essential spectrum, the essential spectrum of a closed operator $S$ being the set of complex numbers $\lambda$ such that $S-\lambda$ is not Fredholm.

For the sake of completeness, we prove the stability of the essential spectrum of the Laplacian acting on forms under perturbations of the metric. As mentioned in 14, Remark 9.10], this result holds on the much weaker hypothesis of regularity of the metric; see Remark A.3. We stay elementary and give a proof in the smooth case. Let $(X, g)$ be a connected, smooth, non-compact Riemannian manifold. Choose $x_{0} \in X$. Let $d_{g}$ be the geodesic distance induced by $g$. Let $\mathscr{H}:=L^{2}\left(X, \Lambda^{*} X, g\right)$ and $\langle\cdot, \cdot\rangle_{g}$ be its scalar product associated to the metric. Since the metric is complete, the operator $D_{g}:=d+\delta_{g}$ is essentially self-adjoint on $\mathcal{C}_{c}^{\infty}\left(X, \Lambda^{*} X\right)$. Its domain is $\mathscr{G}:=\mathcal{D}(d) \cap \mathcal{D}\left(\delta_{g}\right)$. The Laplacian in $\mathscr{H}$ is $\Delta_{g}:=D^{2}$.

Consider some metric $g^{\prime}$ on $X$ satisfying

(A.1) $\alpha(x) g_{x} \leq g_{x}^{\prime} \leq \beta(x) g_{x}$, where $\alpha(x)$ and $\beta(x)$ tend to 1 , as $d_{g}\left(x, x_{0}\right) \rightarrow \infty$.

We denote with the subscript $g^{\prime}$ the object obtained under the metric $g^{\prime}$. From (A.1), we infer that there is a unique $a \in \mathcal{B}(\mathscr{H}), a \geq c>0$, such that $\langle u, v\rangle_{g^{\prime}}=$ $\langle u, a v\rangle_{g}$ for all $u, v \in \mathscr{H}$. We now suppose additionally that $a$ is in $\mathcal{C}^{1}(D)$, meaning there is $C$ so that:

$$
\left|\langle D f, a g\rangle_{g}-\langle a f, D g\rangle_{g}\right| \leq C\|f\|_{g}\|g\|_{g} \text {, for all } f, g \in \mathscr{G} \text {. }
$$

Since $a$ is invertible, one easily obtains that $a^{-1} \in \mathcal{C}^{1}(D)$ and $a^{ \pm 1} \mathscr{G} \subset \mathscr{G}$.

Proposition A.1. Under the Assumptions (A.1) and (A.2), the Laplace operators $\Delta_{g}$ and $\Delta_{g^{\prime}}$, acting on forms, have the same essential spectra.

Proof. Using Assumptions (A.1) and (A.2) and Rellich-Kondrakov, we get

$$
a^{ \pm 1}-1 \in \mathcal{K}(\mathscr{G}, \mathscr{H}) .
$$

Consider $D_{g^{\prime}}:=d+a^{-1} \delta a$. Thanks to (A.3), it is self-adjoint in $\mathscr{H}_{g^{\prime}}$ with domain $\mathscr{G}_{g^{\prime}}:=\mathscr{G}$. Note that $\Delta_{g^{\prime}}:=\left(D_{g^{\prime}}\right)^{2}$. Now remark that the essential spectrum of the closed operator $\Delta_{g^{\prime}}$ does not depend on the choice of the scalar product on $\mathscr{H}_{g^{\prime}}$. We look at it in $\mathscr{H}$, where it is no longer symmetric. Therefore, in order to show that $\Delta_{g}$ and $\Delta_{g^{\prime}}$ have the same essential spectrum, it is enough to show that

$$
\left(\Delta_{g}+1\right)^{-1}-\left(\Delta_{g^{\prime}}+1\right)^{-1} \in \mathcal{K}(\mathscr{H}) .
$$

Now observe that $(\Delta .+1)^{-1}=(D .+i)^{-1}(D .-i)^{-1}$. Hence, it is enough to show that

$$
\left(D_{g} \pm i\right)^{-1}-\left(D_{g^{\prime}} \pm i\right)^{-1} \in \mathcal{K}(\mathscr{H}) .
$$

This follows from the second part of (A.3), since:

$$
\left(D_{g} \pm i\right)^{-1}-\left(D_{g^{\prime}} \pm i\right)^{-1}=-\left(D_{g} \pm i\right)^{-1}\left(a^{-1} \delta(a-1)+\left(a^{-1}-1\right) \delta\right)\left(D_{g^{\prime}} \pm i\right)^{-1} \text {. }
$$

Indeed, for the first term, we have $(a-1) \in \mathcal{K}(\mathscr{G}, \mathscr{H})$ and $a^{-1} \mathscr{G}^{*} \subset \mathscr{G}^{*}$. Therefore, we get $a^{-1} \delta(a-1) \in \mathcal{K}\left(\mathscr{G}, \mathscr{G}^{*}\right)$. For the second term, note that $\left(a^{-1}-1\right) \in$ $\mathcal{K}\left(\mathscr{H}, \mathscr{G}^{*}\right)$.

Remark A.2. The condition (A.2) follows from the stronger

$$
\left|\left\langle d^{*} f, a g\right\rangle_{g}-\left\langle a^{*} f, d g\right\rangle_{g}\right| \leq C\|f\|_{g}\|g\|_{g}, \text { for all } f \in \mathcal{D}\left(d^{*}\right), g \in \mathcal{D}(d) .
$$


In particular for a conformal transformation $g^{\prime}=(1+\rho) g$ with $d \rho$ in $L^{\infty}$, Assumption (A.4) is fulfilled. Note also that (A.4) implies that $a^{ \pm 1}$ stabilizes $\mathcal{D}(d)$ and $\mathcal{D}(\delta)$.

Remark A.3. For Laplacians acting on functions, the condition (A.2) is automatically fulfilled. Moreover, one may treat measurable metrics; see [14, Section 9]. We refer to [16, Lemma 6.3] for similar results concerning perturbations of a magnetic field.

\section{ACKNOWLEDGEMENTS}

We acknowledge useful discussions with Vladimir Georgescu and Andreas Knauf. We are also grateful to the anonymous referee for useful comments.

\section{REFERENCES}

1. W. Amrein, A. Boutet de Monvel and V. Georgescu, $C_{0}$-Groups, commutator methods and spectral theory of N-body Hamiltonians, Birkhäuser, Basel-Boston-Berlin, 1996. MR 1388037 (97h:47001)

2. F. Antoci, On the absolute spectrum of the Laplace-Beltrami operator for p-forms for a class of warped product metrics, Math. Nachr. 279, No. 16, 1749-1772 (2006). MR2274831 (2008h:58054)

3. - On the spectrum of the Laplace-Beltrami operator for p-forms for a class of warped product metrics, Adv. Math. 188 (2004), no. 2, 247-293. MR2087228 (2005d:58052)

4. R. Benedetti and C. Petronio, Lectures on hyperbolic geometry, Universitext, Springer-Verlag, Berlin, 1992. MR.1219310 (94e:57015)

5. C. Bär, The Dirac operator on hyperbolic manifolds of finite volume, J. Differential Geom. 54 (2000), 439-488. MR1823312 (2002c:58048)

6. L. Bueler, The heat kernel weighted Hodge Laplacian on non-compact manifolds, Trans. Amer. Math. Soc., Volume 351, no. 2, 683-713 (1999). MR1443866 (99d:58164)

7. M. Braverman, O. Milatovic and M. Shubin, Essential self-adjointness of Schrödinger-type operators on manifolds, J. Russ. Math. Surv. 57, No. 4, 641-692 (2002); transl. from Usp. Mat. Nauk 57, No. 4, 3-58 (2002). MR1942115 (2004g:58021)

8. G. Carron, $L^{2}$-harmonic forms on non-compact Riemannian manifolds, Proc. Centre Math. Appl. Austral. Nat. Univ. 40, 49-59 (2002). MR1953479 (2003j:58001)

9. H. Donnelly and P. Li, Pure point spectrum and negative curvature for noncompact manifolds, Duke Math. J. 46 (1979), no. 3, 497-503. MR.544241 (80j:35075)

10. R. Froese and P. D. Hislop, Spectral analysis of second-order elliptic operators on noncompact manifolds, Duke Math. J. 58 (1989), No. 1, 103-129. MR.1016416 (90k:58234)

11. M. P. Gaffney, Hilbert space methods in the theory of harmonic integrals, Trans. Amer. Math. Soc., Volume 78, 426-444. (1955) MR0068888 (16:957a)

12. V. Georgescu and C. Gérard, On the Virial Theorem in Quantum Mechanics, Commun. Math. Phys. 208, No.2, 275-281 (1999). MR1729087 (2001b:47134)

13. V. Georgescu, C. Gérard and J. Møller, Commutators, $C_{0}$-semigroups and resolvent estimates, J. Funct. Anal. 216, No. 2, 303-361 (2004). MR2095686 (2005h:47044)

14. V. Georgescu and S. Golénia, Decay preserving operators and stability of the essential spectrum, J. Oper. Theory. 59 (2008), no. 1, 115-155. MR2404467(2009b:47020)

15. S. Golénia and S. Moroianu, The spectrum of magnetic Schrödinger operators and $k$-form Laplacians on conformally cusp manifolds, unpublished manuscript math.DG/0507443.

16. _ Spectral analysis of magnetic Laplacians on conformally cusp manifolds, Ann. H. Poincaré 9 (2008), 131-179. MR2389893 (2009b:58069)

17. F-Z. Gong and F-Y. Wang, On Gromov's theorem and $L^{2}$-Hodge decomposition, Int. J. Math. Math. Sci. 2004, No. 1-4, 25-44 (2004). MR2038792 (2005c:58055)

18. L. Guillopé, Introduction à l'invariant $\eta$, Sémin. Théor. Spectrale Géom. 7, 103-114 (1989).

19. _ Théorie spectrale de quelques variétés à bouts, Ann. Sci. Ecole Norm. Sup. 22, nr. 4 (1989), 137-160. MR985859 (90g:58136)

20. D. Häfner, Sur la théorie de la diffusion pour l'équation de Klein-Gordon dans la métrique de Kerr, Diss. Math. 421 (2003). MR2031494 (2004m:58047) 
21. W. Hantzsche and H. Wendt, Dreidimensionale euklidische Raumformen, Math. Ann. 110 (1935), no. 1, 593-611. MR1512956

22. H. Kumura, Limiting absorption principle and absolute continuity of the Laplacian on a manifold having ends with various radial curvatures, preprint math/0606125.

23. R. Lauter and V. Nistor, On spectra of geometric operators on open manifolds and differentiable groupoids, Electron. Res. Announc. Amer. Math. Soc. 7 (2001), 45-53. MR 1852899 (2002g:58023)

24. M. Lesch and N. Peyerimhoff, On index formulas for manifolds with metric horns, Comm. Partial Diff. Equ. 23 (1998), 649-684. MR1620597 (99d:58166)

25. D. D. Long and A. W. Reid, On the geometric boundaries of hyperbolic 4-manifolds, Geom. Topol. 4 (2000), 171-178. MR1769269 (2001e:57037)

26. J. Lott, On the spectrum of a finite-volume negatively-curved manifold, Amer. J. Math. 123 (2001), no. 2, 185-205. MR1828220 (2002f:58054)

27. R. Mazzeo, The Hodge cohomology of a conformally compact metric, J. Differ. Geom. 28, No. 2, 309-339 (1988). MR961517(89i:58005)

28. R. Mazzeo and R. Phillips, Hodge theory on hyperbolic manifolds, Duke Math. J. 60 (1990), no. 2, 509-559. MR.1047764 (91m:58006)

29. R.B. Melrose, The eta invariant and families of pseudodifferential operators. Math. Res. Letters 2 (1995), 541-561. MR1359962 (96h:58169)

30. R. B. Melrose and V. Nistor, Homology of pseudodifferential operators I. Manifolds with boundary, unpublished manuscript, funct-an/9606005.

31. S. Moroianu, K-Theory of suspended pseudo-differential operators, K-Theory 28 (2003), 167-181. MR.1995875 (2005a:58041)

32. W Weyl laws on open manifolds, Math. Annalen 340 (2008), 1-21. MR 2349766 (2009a:58028)

33. E. Mourre, Absence of singular continuous spectrum for certain selfadjoint operators, Comm. Math. Phys. 78 (1981) 391-408. MR603501 (82c:47030)

34. B. E. Nimershiem, All flat three-manifolds appear as cusps of hyperbolic four-manifolds, Topology Applications 90, no. 1-3 (1998), 109-133. MR1648306 (99i:57029)

35. V. Nistor, Pseudodifferential operators on non-compact manifolds and analysis on polyhedral domains, Spectral geometry of manifolds with boundary and decomposition of manifolds, 307-328, Contemp. Math., 366, Amer. Math. Soc., Providence, RI, 2005. MR 2114493 $(2005 \mathrm{k}: 58050)$

36. F. Pfäffle, The Dirac spectrum of Bieberbach manifolds, J. Geom. Phys. 35 (2000), no. 4, 367-385. MR1780760 (2002b:58045)

37. J. G. Ratcliffe and S. T. Tschantz, The volume spectrum of hyperbolic 4-manifolds, Experiment. Math. 9 (2000), no. 1, 101-125. MR1758804 (2001b:57048)

38. M. Reed and B. Simon, Methods of modern mathematical physics II: Fourier analysis, selfadjointness, Academic Press, New York - San Francisco - London, 1975. MR0493420 $(58: 12429 \mathrm{~b})$

39. B. Simon, Some quantum operators with discrete spectrum but classically continuous spectrum, Ann. Phys. 146 (1983), 209-220. MR701264 (85d:35089)

40. J. A. Wolf, Spaces of constant curvature, Publish or Perish, Inc., Houston, TX, 1984. MR928600 (88k:53002)

Mathematisches Institut der Universität Erlangen-NÜrnberg, Bismarckstr. 1 1/2, 91054 Erlangen, Germany

E-mail address: golenia@mi.uni-erlangen.de

Current address: Institut de Mathématiques de Bordeaux, Université Bordeaux 1, 351 cours

de la Libération, F-33405 Talence cedex, France

E-mail address: Sylvain.Golenia@u-bordeaux1.fr

Institutul de Matematică Al Academiei Române, P.O. Box 1-764, RO-014700 Bucharest, ROMANIA

E-mail address: moroianu@alum.mit.edu 